\title{
Identifying Novel Glioma-Associated Noncoding RNAs by Their Expression Profiles
}

\author{
Alenka Matjašičc, ${ }^{1}$ Mojca Tajnik, ${ }^{1}$ Emanuela Boštjančič, ${ }^{1}$ Mara Popović, ${ }^{2}$ Boštjan Matos, ${ }^{3}$ and \\ Damjan Glavač ${ }^{1}$ \\ ${ }^{1}$ Department of Molecular Genetics, Institute of Pathology, Faculty of Medicine, University of Ljubljana, Ljubljana, Slovenia \\ ${ }^{2}$ Institute of Pathology, Faculty of Medicine, University of Ljubljana, Ljubljana, Slovenia \\ ${ }^{3}$ Department of Neurosurgery, University Medical Centre, Ljubljana, Slovenia
}

Correspondence should be addressed to Damjan Glavač; damjan.glavac@mf.uni-lj.si

Received 12 May 2017; Accepted 20 July 2017; Published 12 September 2017

Academic Editor: Massimo Romani

Copyright @ 2017 Alenka Matjašič et al. This is an open access article distributed under the Creative Commons Attribution License, which permits unrestricted use, distribution, and reproduction in any medium, provided the original work is properly cited.

\begin{abstract}
Long noncoding RNAs (lncRNAs) and microRNAs (miRNAs) play a significant role in cancer development as regulators of protein-coding genes. Their dysregulation was in some extent already associated with glioma, the most aggressive primary brain tumours in adults. The correct diagnosis and treatment selection due to high tumour heterogeneity might be difficult and inadequate, resulting in poor prognosis. Studies of expression patterns of noncoding RNAs (ncRNAs) could provide useful insight in glioma molecular development. We used the qPCR approach to screen and investigate the expression of lncRNAs that were previously deregulated in other cancer types. The study showed altered expression levels for numerous lncRNAs across histologically different glioma samples. Validation of few lncRNAs showed association of expression levels with histological subtype and/or malignancy grade. We also observed deregulated and subtype-distinctive expression for four lncRNA-associated miRNAs. Expression of few lncRNAs and miRNA was also associated with patients' survival, showing potential prognostic value. Several ncRNAs, some already related to glioma and some, to the best of our knowledge, investigated for the first time, might be of greater importance in glioma molecular development and progression. Finding the subtype-specific lncRNA and/or miRNA expression patterns may contribute additional information for a more objective classification.
\end{abstract}

\section{Introduction}

Long noncoding RNAs (lncRNAs) are a group of noncoding RNAs (ncRNAs), classified by the length longer than $200 \mathrm{bp}$ ranging up to $100 \mathrm{~kb}$, and do not appear to have coding potential $[1,2]$. Based on their loci of origin, lncRNAs can be classified as intergenic/intronic, sense/antisense, bidirectional, or overlapping with protein-coding genes or other ncRNAs $[3,4]$. Their lack of coding potential does not mean lack of function; their role in cancer development and progression was already confirmed, although mechanisms of their function are still not fully understood [5] and have yet to be investigated in detail. With progression in expression profiling, tens of functional examples have arisen that associate lncRNAs with diverse range of cellular processes $[2,3]$. Mechanistically, various $\operatorname{lncRNAs}$ act as the cotranscriptional regulators of protein-coding genes [6] and are involved in various biological and developmental pathways, including the acquisition and maintenance of cell characteristics [7]. Certain lncRNAs were found to be closely associated with initiation, proliferation, invasion, and recurrence of glioma and may therefore be exploited for the purposes of subclassification, diagnosis, and prognosis [8]. Genetic and epigenetic changes that permit the survival of abnormal cells and formation of tumour mass often include genes that control critical biological processes and pathways, such as cell proliferation, adhesion, migration, and differentiation [9]. In tumour, deregulated cell proliferation is a critical event required for tumour to grow and invade, that is, for tumour progression [10]. Regulatory $\operatorname{lncRNAs}$ can intercede both tumour suppressor and oncogenic effects $[3,5,11]$, and their aberrant expression emphasizes their role in variety of diseases [5, 12]. Altered expression of certain cancer typespecific lncRNAs can reflect disease progression [13] and 
might serve as potential biomarkers [11, 12]. Another interesting and also the most characterized group of ncRNAs are microRNAs (miRNAs), small, $\sim 22$-nucleotide long ncRNAs, which negatively regulate the expression of mRNA by binding on the untranslated mRNA and preventing their translation $[14,15]$. In addition to lncRNAs, miRNAs can also function as tumour-suppressors or oncogenes. They can be expressed as single genes, gene clusters, or from introns of protein-coding as well as nonprotein-coding genes such as lncRNAs [16].

lncRNAs are widely expressed in mammalian nervous system [7], and several were identified to be specifically linked with neuro-oncological disorders [4, 17], including tumours of central nervous system (CNS) [7]. Glioma are the most common form of primary brain tumours in adults, and despite the progress in therapy, the prognosis of patients, especially those with glioblastoma (GBM), remains ruthless [18]. The lack of effective drugs and lack of inefficient treatment are probably consequences of glioma's heterogeneous and invasive nature. Regarding the latter, the exact pathways of glioma biogenesis and progression are thus still in question [19]. Numerous studies underline the association of changed expression profiles of specific lncRNAs with tumour histological grade $[1,3]$. Aberrant lncRNA expression affects the expression of the target gene, which in turn leads to cell dysfunction and disease progression [2, 20], and may have a direct role in driving the disease state [3]. Indeed, altered expression of lncRNAs and miRNAs has been correlated with development of various cancer types and they could also help elucidate the mechanisms underlying glioma malignant transformation $[12,13]$. In addition, as some glioma subtypes are known to have distinct molecular features [21], expression analyses of coding and noncoding genes can also provide additional information to further distinguish them (classification biomarkers) [13]. Only a few years ago, the golden standard for glioma classification has based mainly on their morphological features, including cells of origin, degree of differentiation, and grade of anaplasia [21]. However, histological diagnoses of glioma tend to be very difficult since many cases are morphologically alike, partly due to the lack of specific (particularly molecular) distinguishable markers [13]. The number of protein-coding genes only differs little between organisms, and as seen, they are not sufficient to properly explain the human gene expression complexity. It is suggested that the expression complexity could be explained right with these noncoding but transcriptionally active genes [22]. Certain lncRNAs, such as H19, MALAT, and linc-POU3F3, have been shown to be involved in cancer progression, and also glioma of different malignancy grades show different patterns of lncRNA expression [22-24]. Also, expression of miRNAs shows biomarker potential, used as diagnostic support or for prognostic and therapeutic application $[25,26]$.

The purpose of this study was to examine the lncRNA expression profile of $90 \operatorname{lncRNAs}$, previously reported to be involved in various types of cancer (Human LncProfiler ${ }^{\mathrm{TM}}$ qPCR Array Kit (System Biosciences, CA, USA)), in glioma samples and to characterize potential glioma-related lncRNAs. Expression of several selected, differentially
TABLE 1: Clinicopathological characteristics of patients in the study.

\begin{tabular}{lcc}
\hline Patients' demographics & & \\
\hline $\begin{array}{l}\text { Number of patients } \\
\text { Sex (female/male) }\end{array}$ & $36 / 28(1.3: 1)$ & \\
Median age at diagnosis & $51.8(3.9-83.1$ years) & \\
(years) (min.-max.) & 29 & \\
Number $<50$ years & 35 & \\
Number $>50$ years & Glioma subtype & WHO grade \\
Glioma classification & 4 diffuse AC & WHO II \\
& 6 anaplastic AC & WHO III \\
& 4 secondary GBM & WHO IV \\
(i) Astrocytoma (AC) & 31 primary GBM & \\
& 14 anaplastic ODG & WHO III \\
(ii) Oligodendroglioma & & \\
(ODG) & 5 anaplastic OAC & WHO III \\
(iii) Oligoastrocytoma & & \\
(OAC) & & \\
Tumour location & 16 (16 na) & \\
(i) Frontal or temporal & & \\
(ii) Other regions & &
\end{tabular}

na: not applicable.

expressed lncRNAs was validated on a bigger number of biological replicates. We searched which of validated lncRNAs might encode, be coexpressed, or coregulated with miRNAs. Those miRNAs were also analysed for potential correlation with their host or coregulated lncRNAs. At last, we evaluated the prognostic value of aberrant ncRNA's expression.

\section{Materials and Methods}

2.1. Tissue Samples and Clinicopathological Data. The study was approved by the National Medical Ethics Committee of the Republic of Slovenia (115/5/14). Sixty-four tumours that were surgically removed from the patients between 2008 and 2012 were chosen for the study. We randomly chose 17 glioma tissue samples for initial expression profiling from our previous glioma data set, with respect to appropriate RNA yield and integrity values. Expression validation set further included 60 glioma tissue samples, of which there were also 13 samples from the initial profiling set.

Tumours were stabilized in RNAlater (Applied Biosystems, USA) immediately after surgical biopsy and incubated at $4^{\circ} \mathrm{C}$ for 7 days. After incubation, samples were stored at $-20^{\circ} \mathrm{C}$ until RNA extraction, as recommended by the manufacturer. All tumours were evaluated by a neuropathologist in order to assess glioma subtype and tumour grade (see Table 1). The tumour biopsies used in the study belonged to 36 female and 28 male patients (median age at diagnosis: 51.8 years \pm SD 16.2). As the expression study was conducted in the year 2012, sample classification is based primarily on a neuropathologist's evaluation of morphological features and previous diagnosis if existed and only in some cases based on the status of p53 and $1 \mathrm{p} / 19 \mathrm{q}$ codeletion. Samples of diffuse and anaplastic astrocytoma were united in one subgroup, that is, grade II + III astrocytoma, for simpler 
comparison with other subtypes. For reference RNA, we used the FirstChoice Human Brain Reference Total RNA (cat. number 6050, Ambion, Invitrogen, USA) (further referred to as reference RNA), obtained from the brain tissue of 23 individuals without any signs of neurodegeneration.

2.2. RNA Extraction from Fresh Tissue. RNA from all the samples used in primary profiling set and validation set was extracted following the same isolation protocol. Total RNA from up to $30 \mathrm{mg}$ of each tissue was isolated using TRIzol reagent (Invitrogen, USA), followed by purification using miRNeasy Mini Kit (Qiagen, Germany), according to the manufacturer's instructions. The yield was measured spectrophotometrically using NanoDrop-1000 (Thermo Scientific, USA), and the quality was evaluated on Bioanalyzer 2100 (Agilent Technologies, USA). After the RNA extraction and quality assessment, the samples with appropriate RNA yield and integrity values (concentration higher than $100 \mathrm{ng} / \mu \mathrm{L}$ and $\mathrm{RIN}>5.5$ ) were considered for expression profiling and further validation analyses.

\subsection{LncRNA Expression Profiling Using Human LncProfiler} qPCR Array Kit. We performed a primary expression profiling of 90 lncRNAs that have already been associated with different types of cancer (Human LncProfiler qPCR Array Kit (System Biosciences, CA, USA)). IncRNA profiling of these 90 lncRNAs was performed using qPCR amplification of cDNA from an initial set of 17 glioma samples of different histopathological characteristics and normal brain reference RNA (control).

First strand of cDNA was synthesized using Human LncProfiler qPCR Array Kit with oligo-dT and random hexamers, according to the manufacturer's instructions. All qPCR reactions were carried out in triplicates and included a negative control. Quantification was performed using the ABI Prism 7900 sequence detection system (Applied Biosystems, USA). Cycling conditions were as follows: 2 minutes at $50^{\circ} \mathrm{C}, 10$ minutes at $95^{\circ} \mathrm{C}$, and 40 cycles of 15 seconds at $95^{\circ} \mathrm{C}$, and one minute at $60^{\circ} \mathrm{C}$. The signal was collected at the endpoint of each cycle. A cohort of five reference genes was simultaneously analysed for the normalization process (18S rRNA, RNU43, GAPDH, LAMIN A/C, and U6 snRNA), and the optimum reference genes were identified using NormFinder algorithm [27]. Expression values of lncRNAs in glioma were compared to values of normal brain reference RNA, and results were statistically analysed.

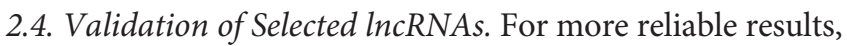
results need to be validated on a larger number of biological replicates. Upon statistical analysis, we selected 7 lncRNAs (EGO-A, 7SL, RNCR3, MEG3, HOTAIR, ZFAS1, and JPX) for validation and to further investigate their differential expression on a cohort of 60 glioma samples and normal brain reference RNA (control). For the subsequent analyses of lncRNA expression, cDNA was generated with Protoscript M-MuLV Taq RT-PCR kit using random primers (New England Biolabs, UK) according to the manufacturer's instructions, except were otherwise indicated. Reverse transcription reactions were prepared in $10 \mu \mathrm{l}$ master mix with
$500 \mathrm{ng}$ of total RNA. Expression analyses were validated using the TaqMan or SybrGreen-based qPCR technology, dependent on probes availability. All qPCR reactions were carried out on Rotor-Gene Q (Qiagen, Germany), and each sample was analysed in duplicate. Thermal conditions were applied as follows: initial denaturation at $95^{\circ} \mathrm{C}$ for 15 minutes and 40 cycles of denaturation at $95^{\circ} \mathrm{C}$ for 15 seconds and annealing at $60^{\circ} \mathrm{C}$ for 1 minute, and following amplification melting curves were acquired. The signal was collected at the endpoint of each cycle.

2.4.1. TaqMan-Based Technology. qPCR reactions for MEG3, $R N C R 3$, JPX, and ZFAS1 were prepared with lncRNA-specific TaqMan assay (MEG3 Assay ID Hs01098508_mi; RNCR3 Assay ID Hs01039195_gi; JPX Assay ID Hs03681129_m1, ZFAS1 Assay ID Hs03300756_m1) (all Applied Biosystems, USA) and FastStart Essential DNA Probes Master Mix (Roche, Germany) or TaqMan Gene Expression Master Mix (Applied Biosystems, USA) in $10 \mu \mathrm{l}$ reaction volume. Reference genes GAPDH and $18 S$ rRNA were analysed in both conditions.

2.4.2. SybrGreen-Based Technology. qPCR reactions for $E G O-A, 7 S L$, and HOTAIR were prepared with designed, IncRNA-specific primers (EGO-A: F - CTTCTCCTCCAGG CCATACC, R - CCATTGTGTAGCCCCG; 7SL: F - CTGT AGTCCCAGCTACTCG, R - CCCGGGAGGTCACCAT ATT; and HOTAIR: F - CAGTGGGGAACTCTGACTCG, R - GTGCCTGGTGCTCTCTTACC) and Power Sybr ${ }^{\circledR}$ Green PCR Master Mix (Applied Biosystems, USA) in $10 \mu \mathrm{l}$ reaction volume. GAPDH and U6 snRNA were used as reference genes.

2.5. Quantitative Real-Time PCR of miRNAs. miRNAs were analysed using either the miScript system (SybrGreen, Qiagen, Germany) or TaqMan-based technology (Applied Biosystems, USA) according to their corresponding lncRNAs. All the reagents were from Qiagen or Applied Biosystems, respectively, except where otherwise indicated. qPCR was carried out using the Rotor-Gene Q (Qiagen, Germany).

2.5.1. miScript System (Qiagen, Germany). All of the steps were performed according to manufacturer's protocol, except where otherwise indicated. miScript reverse transcription kit was used for RT in a $10 \mu \mathrm{l}$ reaction master mix with $100 \mathrm{ng}$ of total RNA. All of the $\mathrm{qPCR}$ reactions were performed in duplicate, and following amplification melting curves were acquired. MicroRNAs $m i R-196 a$ and $m i R-125 b$ were tested relatively to RNU6B and SCARNA17 (RNU6B, SCARNA17, $S N O R A 73 A$, and RNU1B were tested as reference genes). The signal was collected at the endpoint of every cycle.

2.5.2. TaqMan-Based Technology. All of the steps were performed according to manufacturer's protocol, except where otherwise indicated. RNU6B, RNU48, RNU58A, and $H Y 3$ were tested as references genes. MicroRNAs $m i R-124 a$ and $m i R-770$ were tested relatively to RNU6B and RNU58A. Briefly, the $10 \mu \mathrm{l}$ RT reaction master mix was performed with 10 ng of total RNA sample. The cDNA was diluted 30-fold, and $4.5 \mu \mathrm{l}$ was used for each $10 \mu \mathrm{l} \mathrm{qPCR}$ reaction. The qPCR 
reactions were performed in duplicates, and the signal was collected at the endpoint of every cycle.

2.6. Computational and Statistical Analysis. Relative quantification of lncRNA and miRNA levels of the target gene was calculated using the $\Delta \Delta \mathrm{C}_{\mathrm{T}}$ and represents the difference between $\Delta \mathrm{C}_{\mathrm{T}}$ tumour RNA and $\Delta \mathrm{C}_{\mathrm{T}}$ reference RNA, normalized to expression of endogenous controls [28]. If $\Delta \Delta \mathrm{C}_{\mathrm{T}}$ was significantly $(2 \sigma)$ higher or lower than zero, the expression was considered to be significantly different. The calculation method was used for computational analyses for both lncRNA profiling and validation of differentially expressed lncRNA and miRNA.

Calculated $\Delta \Delta \mathrm{C}_{\mathrm{T}}$ values and the $\mathrm{R}$ programming language were used for unsupervised hierarchical clustering analysis, with Pearson correlation for distance metric. All statistical tests were performed using the IBM SPSS Statistics 20. software (IBM Corporation, New York, USA). Not all cases had all data available. Factor analysis was performed using the principal component extraction method and the direct oblimin as the rotation method. We used the Mann-Whitney 2 -independent samples test to determine which of the $90 \operatorname{lncRNAs}$ are differentially expressed, and results are graphically presented as log values on the heat map. For determining significant differences in expression (using $\Delta \Delta \mathrm{C}_{\mathrm{T}}$ ) of lncRNAs and miRNAs between glioma subtypes, we used the Kruskal-Wallis $k$-independent test (multiple comparisons). Mann-Whitney 2-independent samples test was performed to cross test expression differences between two subtypes. The expression differences were considered statistically significant when the differences in tested groups reached or were below $p \leq 0.05$. The relative quantification of lncRNA and miRNA levels is graphically presented as average fold change $\left(2^{-\Delta \Delta C T}\right)$. The Pearson's correlation coefficient was used to define expression relationships and Kaplan-Meier estimate for constructing survival curves (analysed by log-rank test). To assess the relative risk for each factor, we performed the univariate and multivariate Cox regression analyses using $\mathrm{R}$ language. We used the $\Delta \Delta \mathrm{C}_{\mathrm{T}}$ values, and two-sided $p$ value $<0.05$ was regarded as significant.

\section{Results}

3.1. Profiling Shows Numerous lncRNAs with Altered Expression. lncRNA profiling revealed changes in expression levels for numerous lncRNA (74/90 lncRNAs analysed) in the primary set of 17 glioma samples compared to normal brain reference RNA (pooled RNA samples from 23 donors, commercially available). Unsupervised hierarchical clustering (Figure 1) upon similarity of gene expression profiles between tumour samples showed roughly three molecular groups; however, gene expression-based groups did not coincide with samples' histological subtypes, suggesting the heterogeneous lncRNA expression background of morphologically similar tumours. Factor analysis, with the principal component extraction method based on 74 gene expression profiles, showed two principle components, which together account for $60.8 \%$ of variance in gene expression (Figure 2).
The component 1 , accountable for $46.8 \%$ of variance, separates samples in two main groups, which remain more or less the same with component 2 (accountable for $13.95 \%$ of variance). Sample grouping again showing differences in lncRNA expression profile vary between glioma subtype and to some extent coincided with unsupervised hierarchical clustering (comparing Figures 1 and 2).

3.2. Expression Validation of Seven IncRNAs. Among differentially expressed lncRNAs, we further validated seven lncRNAs (7SL, EGO-A, HOTAIR, JPX, MEG3, RNCR3, and ZFAS1) on a bigger cohort of glioma samples of different WHO malignancy grades and histopathological subtypes. Expression of lncRNAs MEG3, JPX, RNCR3, and ZFAS1 significantly differed between low and high malignancy grade (Mann-Whitney test; $\Delta \Delta \mathrm{C}_{\mathrm{T}}$ values) (Figure 3). MEG3, JPX, $R N C R 3$, and ZFAS1 showed significant decrease with tumour malignant progression.

Next, we grouped the samples upon their histological subtype into five groups and compared lncRNA's expression between all five using the Kruskal-Wallis test ( $\Delta \Delta \mathrm{C}_{\mathrm{T}}$ values). lncRNA expression patterns for corresponding glioma subtype are graphically presented in Figure 4 . We observed significant differences across all samples for each of the IncRNAs. Expression of HOTAIR and ZFAS1 was found overexpressed, and MEG3, JPX, and RNCR3 were substantially underexpressed. EGO-A showed overexpression in all subtypes, except in secondary GBMs. Expression of 7SL was found near normal level in oligodendroglioma and oligoastrocytoma, but underexpressed in astrocytoma, primary, and secondary GBMs.

3.3. There Are Distinctive IncRNA Expression Patterns between Glioma Subtypes. Kruskal-Wallis test does not tell between which subgroups the differences in expression significantly differ. Using the Mann-Whitney test, we compared the $\Delta \Delta \mathrm{C}_{\mathrm{T}}$ values of specific IncRNA of histologically determined subtypes among each other to establish subtype expression (Table 2) (for all graphic comparisons see graphs on Figure 4). HOTAIR exhibited much higher expression in oligodendroglioma and oligoastrocytoma compared to astrocytic tumours. ZFAS1 was overexpressed in oligodendroglioma and oligoastrocytoma when cross tested with primary GBMs, which showed near normal expression levels. MEG3 and JPX expression showed especially lower levels in primary GBMs compared to other astrocytic glioma. We also observed similar pattern for RNCR3 with much lower expression levels in GBMs. And as observed before, expression of MEG3, JPX, and RNCR3 decreases with malignancy grade (Figure 3). Significant differences were also found for $7 S L$ expression between oligodendroglioma and astrocytoma. Especially different pattern was observed for $E G O-A$ in secondary GBMs.

3.4. Changed Expression of IncRNA-Related miRNA. In the subset of significantly changed lncRNAs we found that four are also encoding miRNAs. 7SL was recently suggested to be regulated by $m i R-125 b$ [29]. Wei et al. also suggest $m i R-125 b$ to be a potential biomarker of glioma [30]. HOTAIR has been 


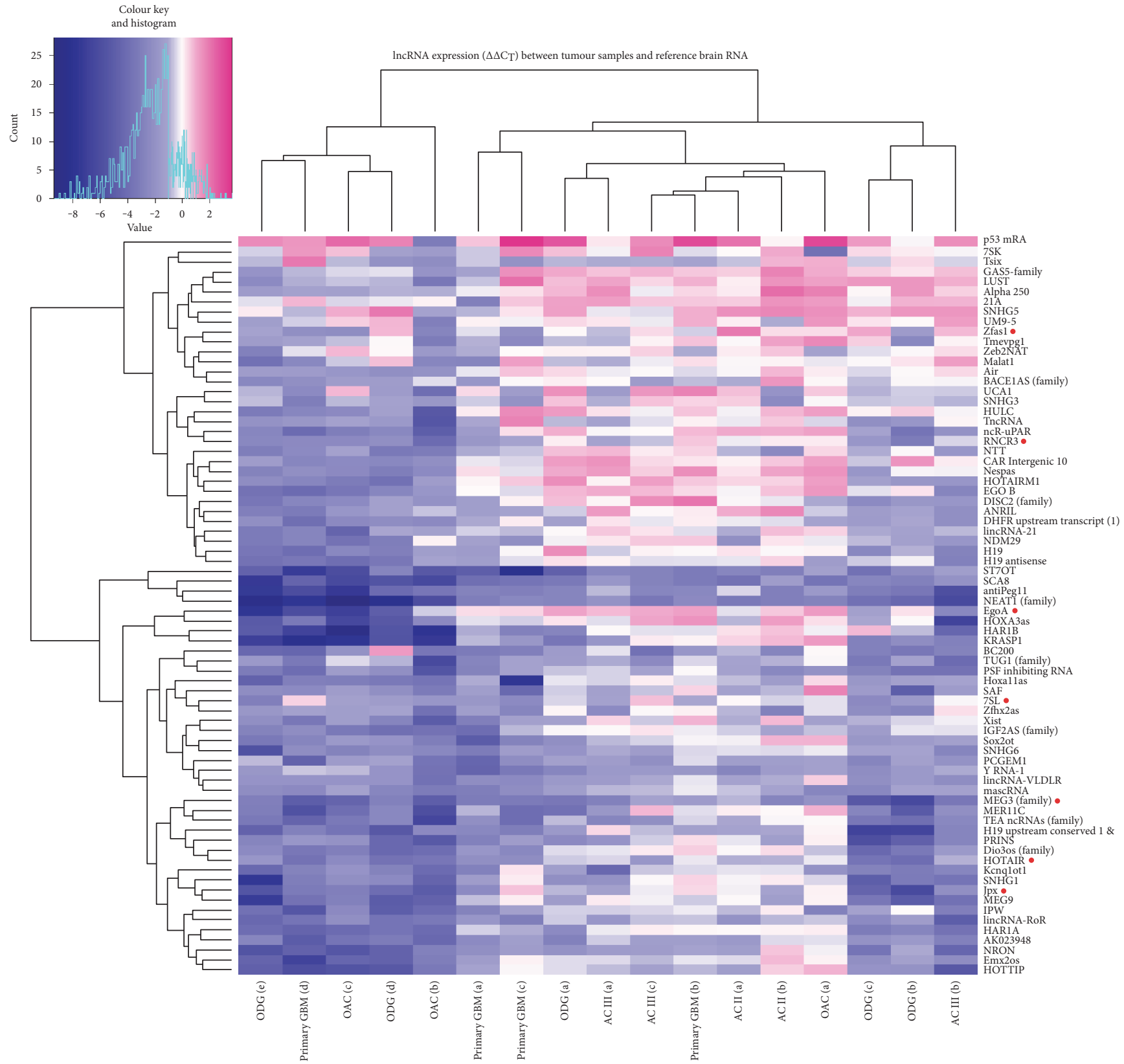

FIGURE 1: Heat map of lncRNA profiling analyses of glioma samples. The figure represents $\Delta \Delta \mathrm{C}_{\mathrm{T}}$ values of differentially expressed lncRNAs in glioma samples compared to human brain reference RNA. Data are presented on a colour scale where shades of blue represent decreased expression and pink as increased expression, with $\Delta \Delta \mathrm{C}_{\mathrm{T}}$ cut-off values set at -1 and 1 . On the top of the figure is presented unsupervised Pearson's hierarchical clustering of samples. AC: astrocytoma of WHO grade II or III; OAC: oligoastrocytoma; ODG: oligodendroglioma. Genes denoted by red dot were selected for further qPCR validation and analysis.

found to be coregulated with $m i R-196 a$ [31], and genes for $R N C R 3$ and MEG3 are acting as host genes for $m i R-124 a$ and $m i R-770$ [32], respectively. We determined the expression levels of these four miRNAs and performed a IncRNA-miRNA linear correlation analysis to determine their possible relationship.

Expression levels of $m i R-124 a$ and $m i R-196 a$ significantly differ between the WHO malignancy grades (Mann-Whitney test; $\Delta \Delta \mathrm{C}_{\mathrm{T}}$ values) (Figure 5). We observed significant differences in expression of miR-770 among all glioma subtypes $(p<0.001)$. It has been decreased in all samples of astrocytoma, oligoastrocytoma, and oligodendroglioma and in majority of GBMs (expression varied). Expression significantly changed in oligodendroglioma and oligoastrocytoma compared to astrocytic subtypes (Figure 6(a)). miR-196a was also decreased in all groups $(p=0.001)$ and was significantly lower in oligodendroglioma, astrocytoma, and oligoastrocytoma compared to primary GBMs (2- to 4-fold) and secondary GBMs (up to 10 -fold). In a proportion ( 36\%) of primary GBMs, miR-196a was slightly overexpressed (Figure 6(a)). For miR-124a, expression was decreased in all glioma subtypes when compared to reference RNA, but there was no significant 


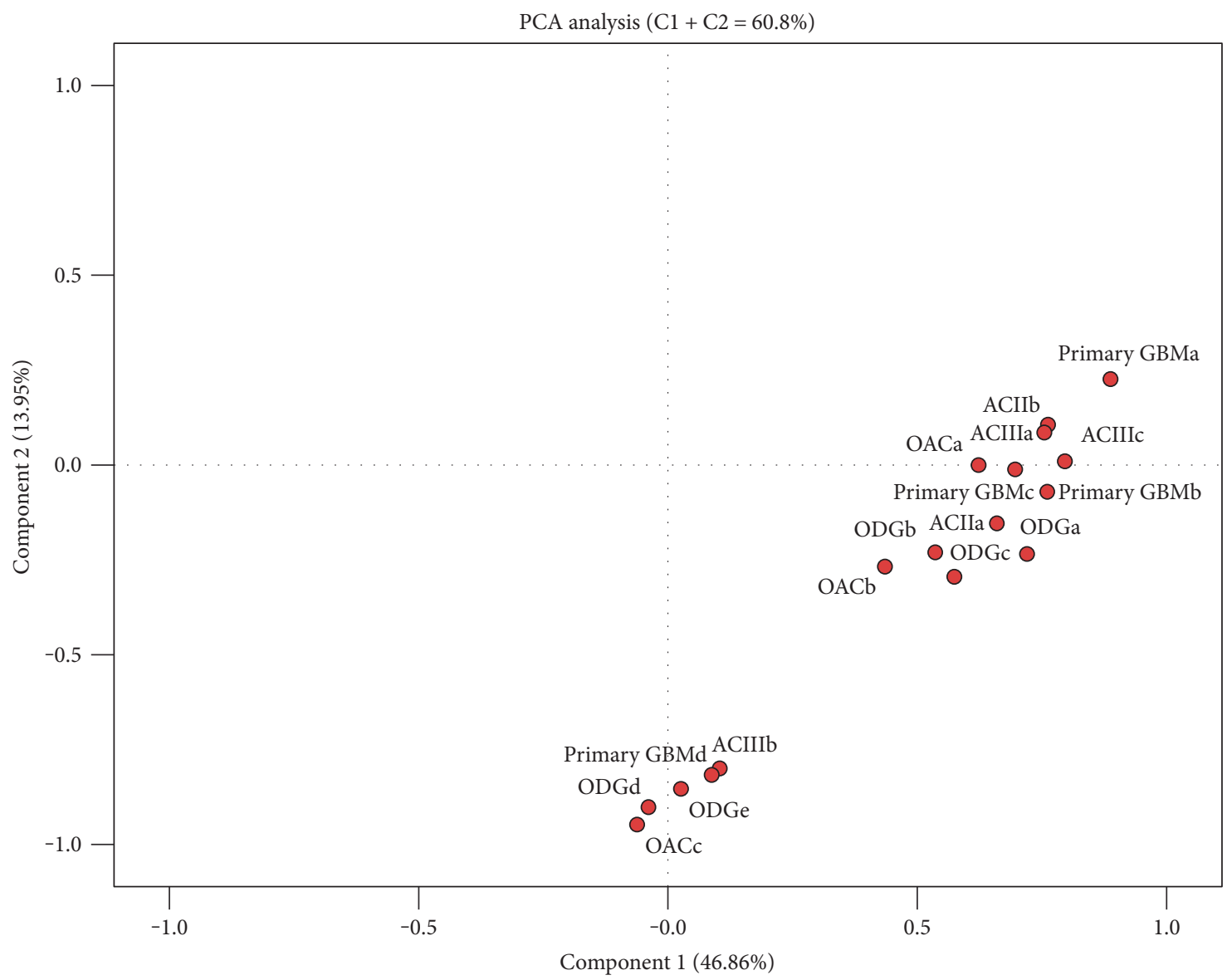

Figure 2: Principal component analysis of individual samples, generated with expression of 74 differentially expressed lncRNAs. AC: astrocytoma of grade II or III; GBMs: glioblastoma; ODG: oligodendroglioma; OAC: oligoastrocytoma. Letters at the end mean a consecutive sample.

change between different glioma subtypes. Also, miR-125b showed underexpression, except in secondary GBMs, for which we observed significant change comparing to other subtypes $(p=0.017$ ) (Figure 6(a)). When comparing expression values of miRNAs between two different glioma subtypes, all miRNAs were shown to be differentially expressed between at least two glioma subtypes, except miR-124a, which did not reach statistically significant difference in expression between any two groups of samples (Figure 6(a)).

We observed moderate negative linear correlation $\left(r_{\mathrm{s}}=-0.373, p=0.005\right)$ between expression of $m i R-770$ and MEG3, and positive correlation $\left(r_{\mathrm{s}}=0.291, p=0.028\right)$ between expression of $m i R-124 a$ and RNCR3 in all glioma samples. Both lncRNAs act as miRNA host genes. miR$125 b$ and $7 S L$ and miR-196a and HOTAIR did not show significant correlation (Figure 6(b)). However, the lncRNAmiRNA relationship results provided are only suggestive as functional studies are required for stronger conclusion.

3.5. ncRNA's Expression Is Associated with Patients Age at Diagnosis and Survival Time. Expression of certain lncRNAs seems to be associated with patient's age at the time of diagnosis as correlation analysis showed moderate negative association for MEG3 ( $\left.r_{\mathrm{s}}=-0.336, p=0.009, R^{2}=0.113\right)$, ZFAS1 $\left(r_{\mathrm{s}}=-0.368, p=0.004, R^{2}=0.164\right)$, and RNCR3 $\left(r_{\mathrm{s}}=-0.431\right.$, $p=0.001, R^{2}=0.186$ ).

Also, miR-196a showed moderate association with patient's age at time of diagnosis $\left(r_{\mathrm{s}}=0.290, p=0.039\right)$.

The Kaplan-Meier estimate showed better survival for patients who were younger than 50 years at the time of diagnosis (Pearson's correlation $r_{\mathrm{s}}=-0.482, p<0.001$ ), but no difference regarding the sex or tumour location (Figure 7). Also, survival significantly differs between astrocytoma, primary GBMs, and oligodendroglioma. Because of a low sample number of oligoastrocytoma and secondary GBMs, we did not include them in the survival plot regarding the subtype $(p=0.001)$ (Figure 7). For determining possible association of gene's differential expression and survival, expression values of ncRNAs analysed were classified as low or high based upon individual $\Delta \Delta \mathrm{Ct}$ mean expression value. Kaplan-Meier plots and further analysis of survival differences (log-rank analysis) showed significant differences in survival for expression levels of MEG3, ZFAS1, RNCR3, miR-770, miR-125b, and miR-196a (Figure 8). These ncRNAs also showed significant association with survival when considering only the expression of astrocytoma, primary GBMs, and oligodendroglioma (data not shown). To identify the potential 

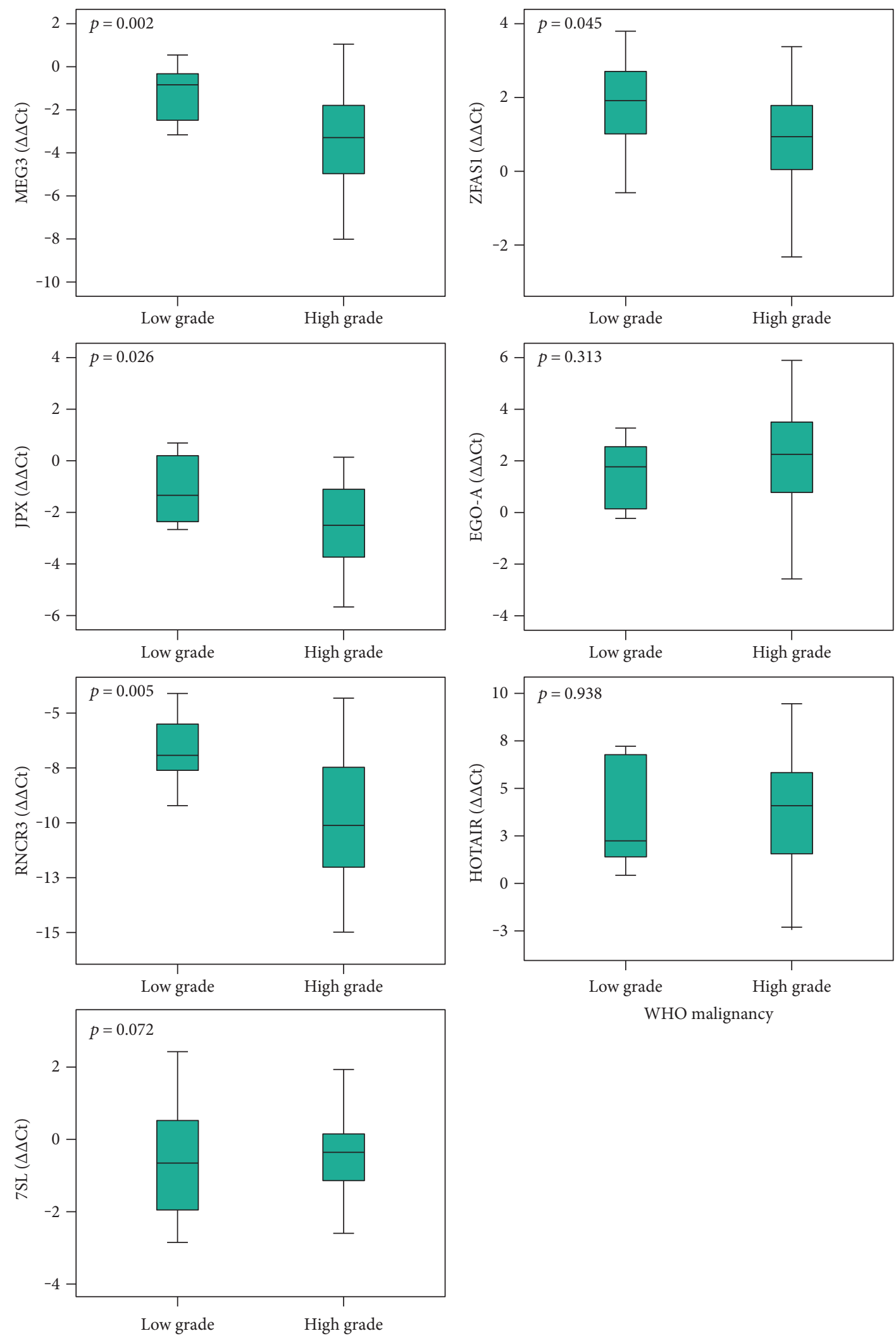

Low grade

High grade

WHO malignancy

WHO malignancy

FIGURE 3: qPCR results of lncRNA gene expression regarding the WHO malignancy grade. Expression analyses of 7 lncRNAs, previously determined as differentially expressed, between low and high malignancy grade. Data are presented as $\Delta \Delta \mathrm{C}_{\mathrm{T}}$ box plots \pm standard error. 

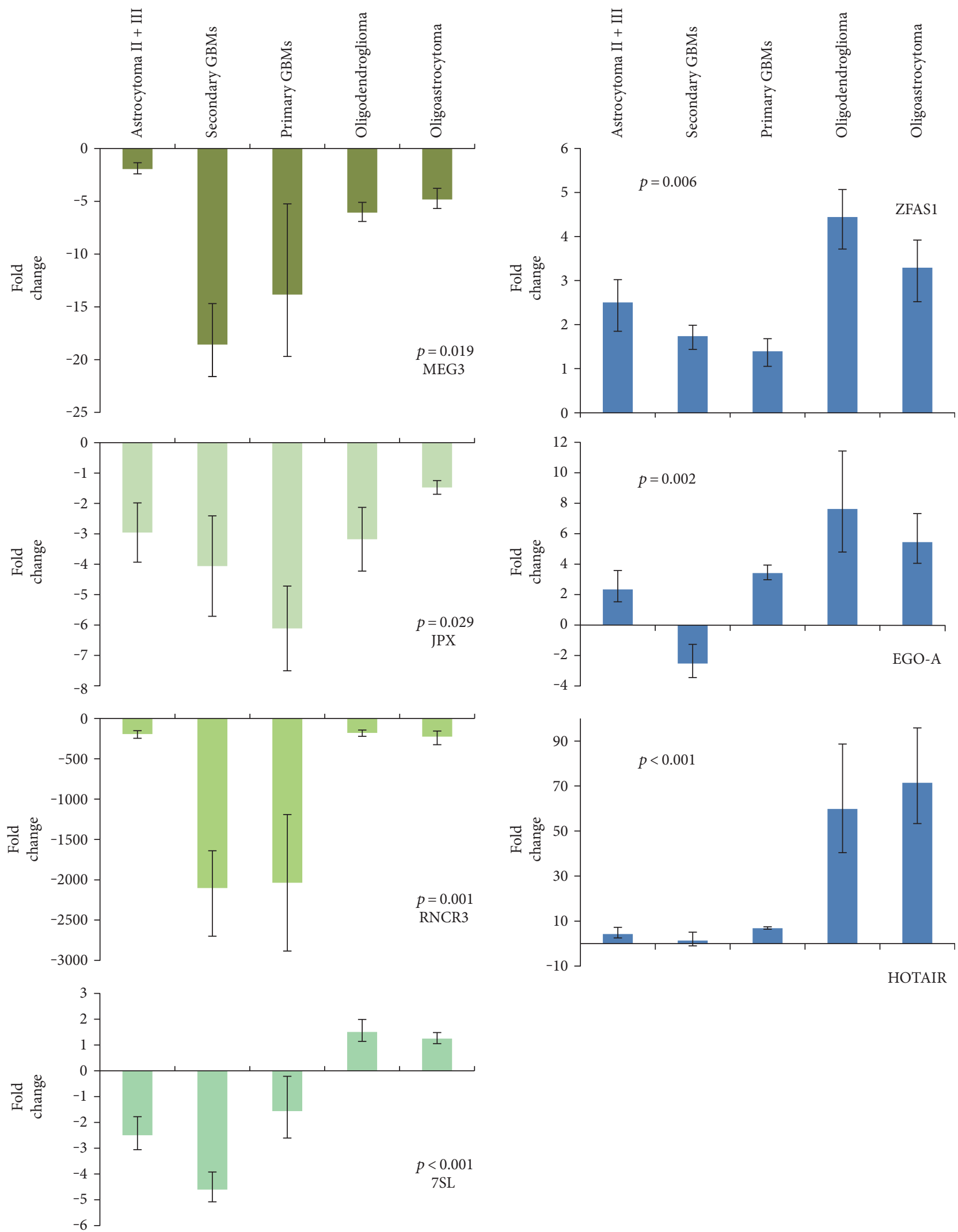

FIGURE 4: qPCR results of lncRNA gene expression in glioma subtypes. Expression analyses of glioma samples and human brain reference RNA for a subset of seven lncRNAs previously determined as differentially expressed. Data are presented as mean fold change (FC) \pm standard FC error. $p$ shows statistical significance for Kruskal-Wallis test of expression differences among all five subgroups (threshold set at $p<0.05$ ). 
TABLE 2: Significantly different expression of lncRNA between glioma subtypes (represented as $p$ value).

Mann-Whitney 2-independent test Secondary glioblastoma Primary glioblastoma Anaplastic oligodendroglioma

Anaplastic oligoastrocytoma

\begin{tabular}{|c|c|c|c|c|}
\hline \multirow[t]{4}{*}{ Grade II + III astrocytoma } & $E G O-A 0.016$ & $\begin{array}{c}R N C R 30.001 \\
M E G 30.001\end{array}$ & $\begin{array}{c}\text { HOTAIR } 0.004 \\
\text { EGO-A } 0.010 \\
\quad 7 S L 0.005\end{array}$ & $\begin{array}{l}E G O-A 0.024 \\
\quad 7 S L 0.038\end{array}$ \\
\hline & Secondary glioblastoma & $\begin{array}{c}\text { HOTAIR } 0.039 \\
\text { EGO-A } 0.006 \\
\quad 7 S L 0.011\end{array}$ & $\begin{array}{c}\text { HOTAIR } 0.003 \\
\text { EGO-A } 0.004 \\
\text { 7SL } 0.003\end{array}$ & $\begin{array}{c}\text { HOTAIR } 0.034 \\
\text { EGO-A } 0.0217 S L 0.021\end{array}$ \\
\hline & & Primary glioblastoma & $\begin{array}{c}\text { HOTAIR } 0.001 \\
\text { RNCR3 } 0.001 \\
\text { ZFAS1 } 0.001 \\
\text { JPX } 0.019 \\
\text { 7SL }<0.001\end{array}$ & $\begin{array}{c}\text { HOTAIR } 0.041 \\
\text { RNCR3 } 0.018 \\
\text { ZFAS1 } 0.036 \\
\text { JPX } 0.014\end{array}$ \\
\hline & & & $\begin{array}{c}\text { Anaplastic } \\
\text { oligodendroglioma }\end{array}$ & l \\
\hline
\end{tabular}
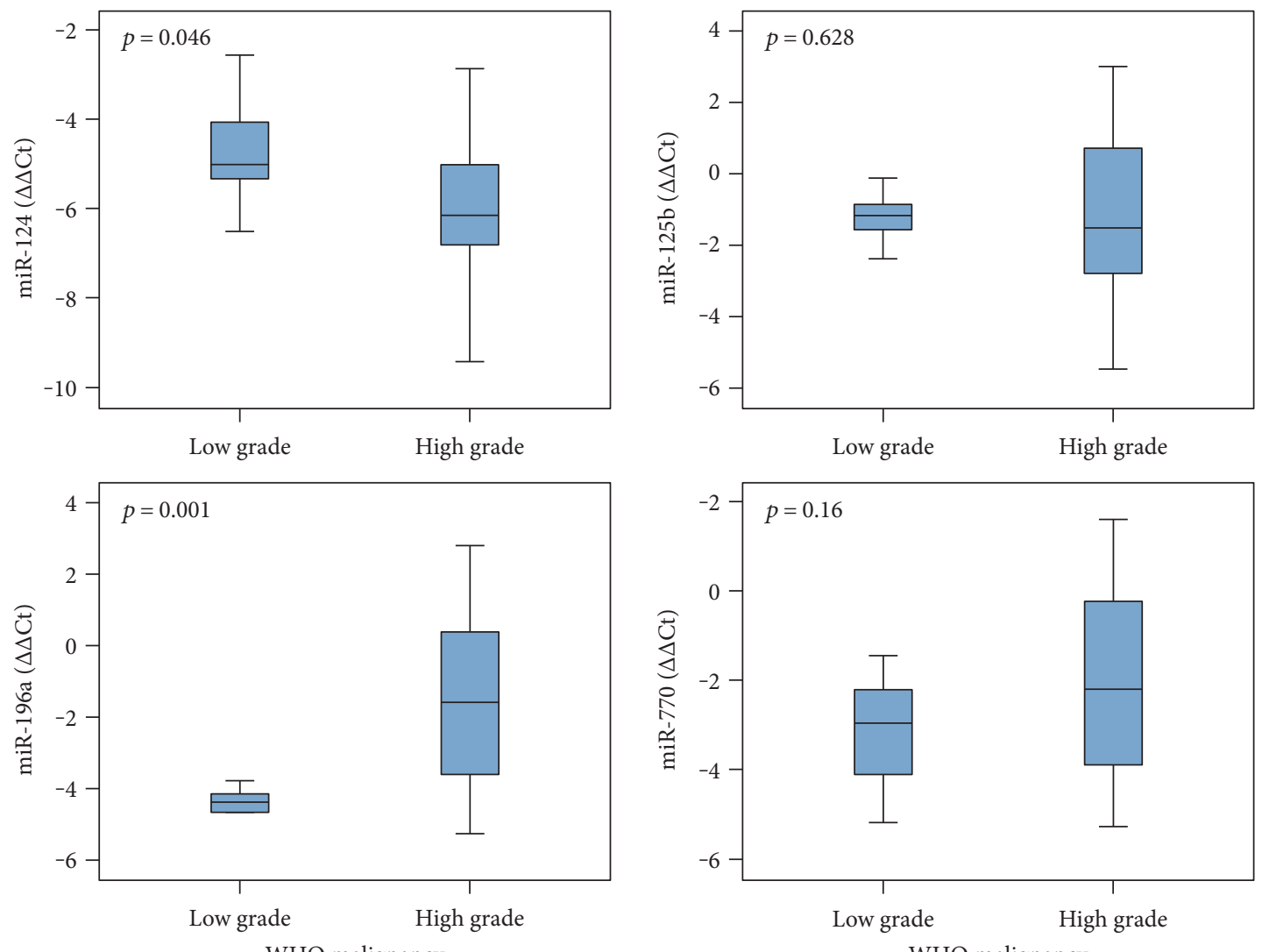

WHO malignancy

FIGURE 5: miRNA expression levels regarding the WHO malignancy grade. Expression analyses of four lncRNA-associated miRNAs between low and high malignancy grade glioma. Data are presented as $\Delta \Delta \mathrm{C}_{\mathrm{T}}$ box plots \pm standard error.

prognostic value of ncRNA expression, we used the univariate Cox proportional hazards regression analysis. We found significant association of survival with patient's age at diagnosis and expression of MEG3, ZFAS1, RNCR3, $m i R-125 b$, $m i R-196 a$, and $m i R-770$, respectively. For the multivariate analysis, we took into consideration only the parameters with $p<0.05$ in the univariate analysis. Results showed patient's age at diagnosis and expression of
ZFAS1, RNCR3, miR-125b, and $m i R-196 a$ as potential independent prognostic variables.

\section{Discussion}

Although many of lncRNAs known are involved in a variety of cancers, only a few have been associated with a particular cancer type [12]. IncRNA expression profiling revealed 

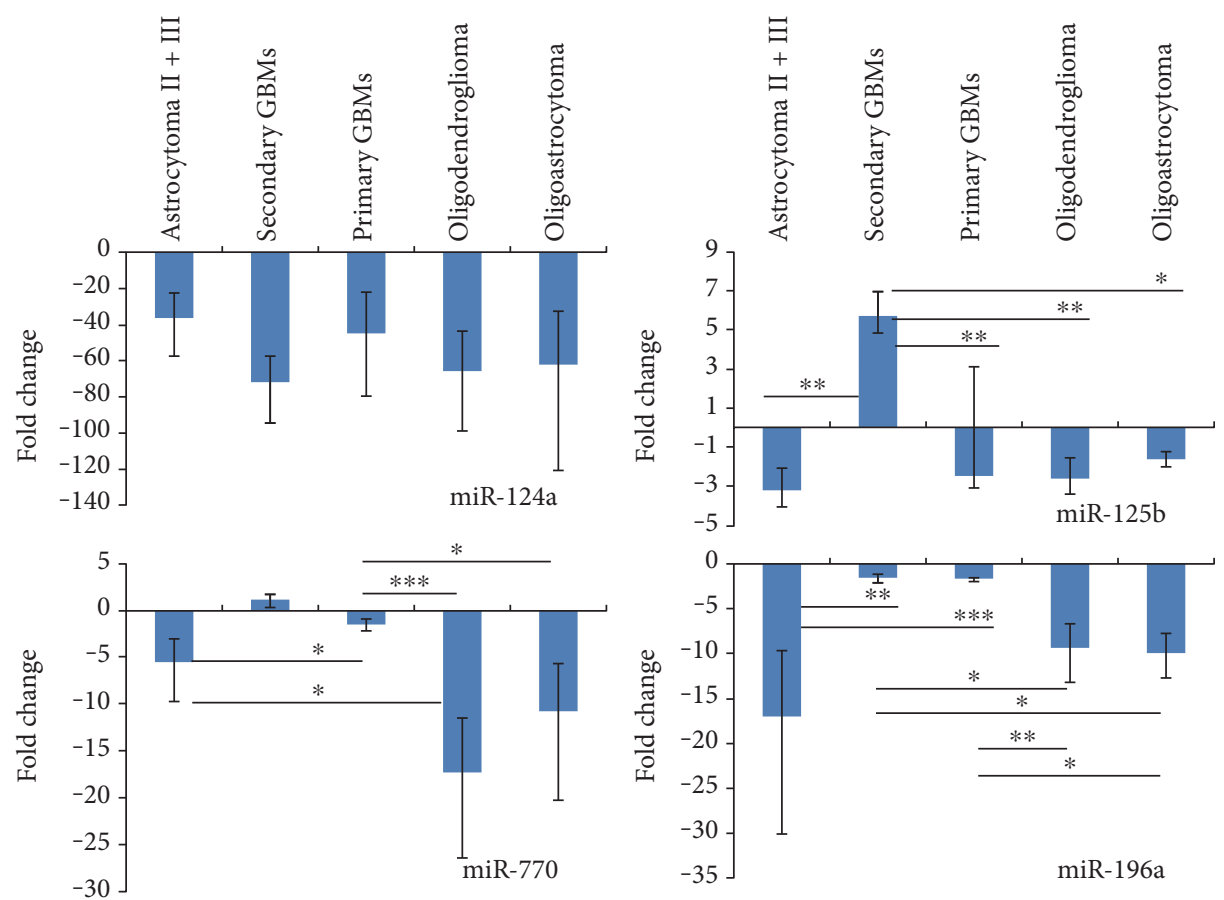

(a)
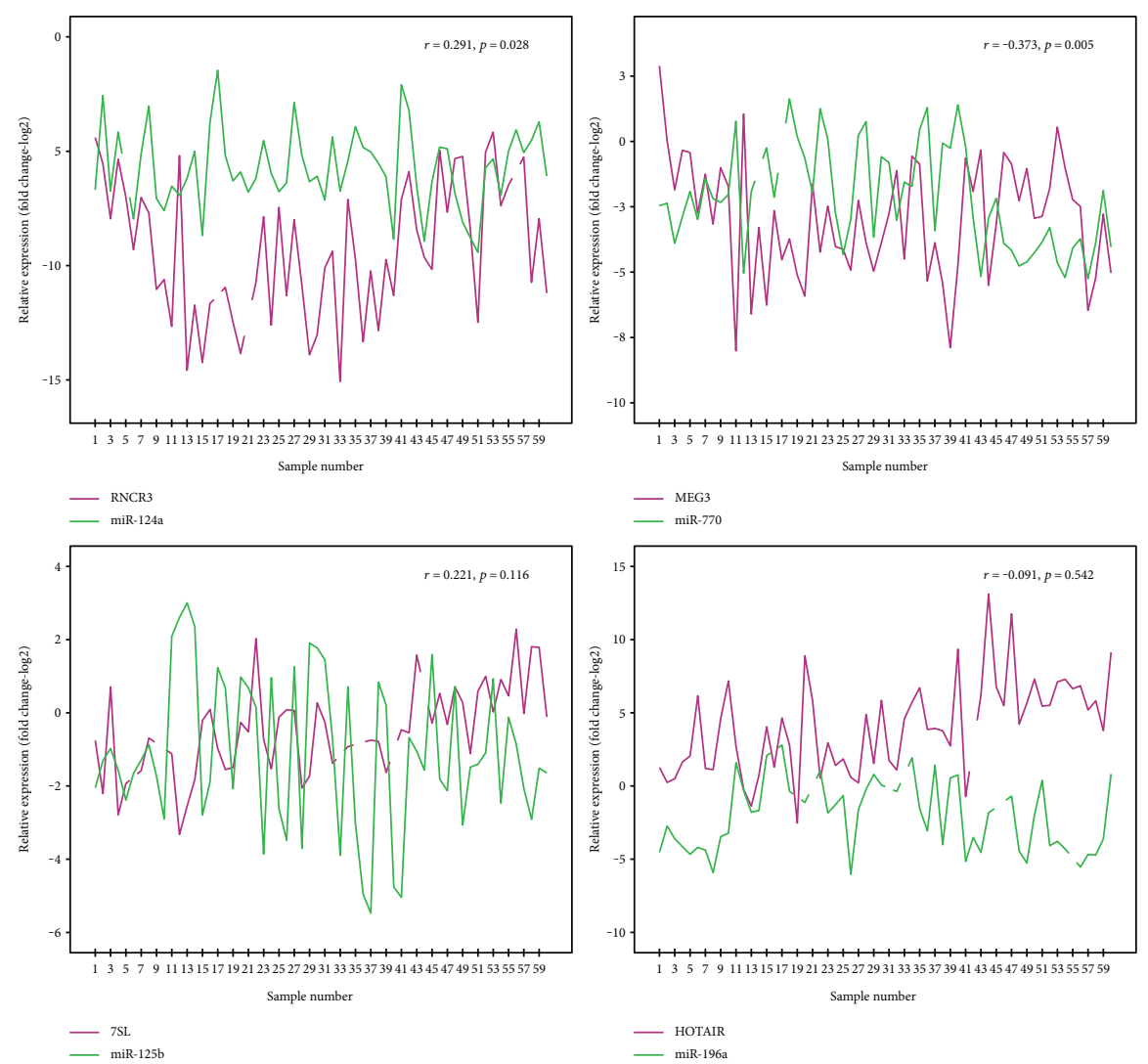

(b)

FIGURE 6: Expression results of miRNAs related to differentially expressed lncRNAs in glioma subtypes. (a) Expression analyses of miRNA, found to be associated with lncRNAs analysed, on the same cohort of sample as for lncRNAs as well as on human brain reference RNA. Data are presented as mean fold change (FC) \pm standard FC error. Bars with asterisks represent significance for in-betweengroup comparison $\left({ }^{*} 0.01 \leq p<0.05 ;{ }^{* *} 0.001 \leq p<0.01 ;{ }^{* * *} p<0.001\right)$. (b) Correlation relationship of expression levels in individual sample for miRNA and its related lncRNA. Letter $r$ represents the magnitude of correlation and $p$ the significance value. 

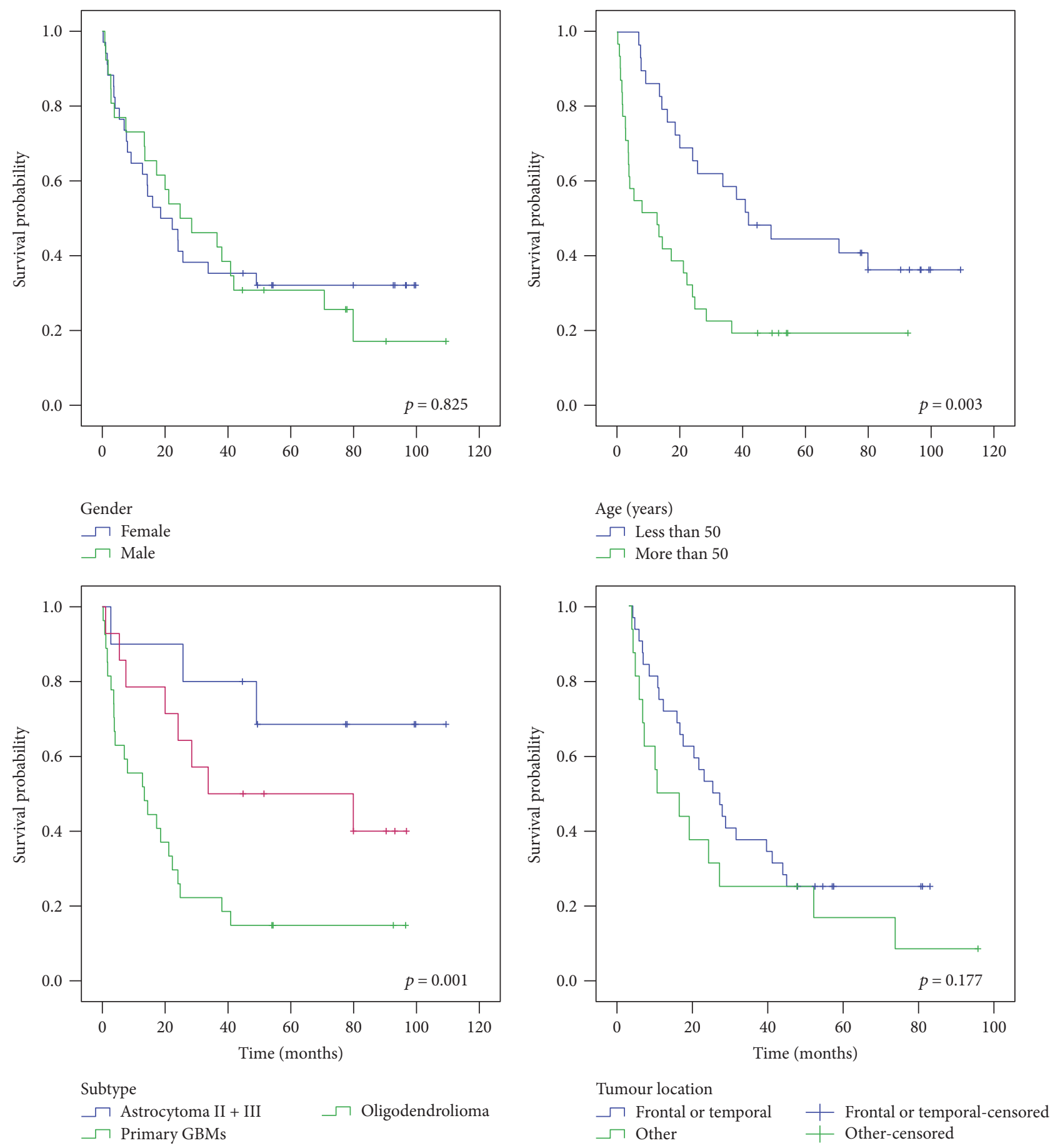

Figure 7: Kaplan-Meier survival curve analysis shows poor prognosis for patients older than 50 years at time of diagnosis and with diagnosed GBM.

numerous lncRNA to be widely expressed in glioma, thus once again acknowledging the complexity of the brain and brain tumours and interweaving of different molecular levels. Tumour sample clustering based on expressional profile of 74 lncRNAs did not coincide with histopathological subtype, which suggests heterogeneous global expression of lncRNAs. However, determining glioma lncRNA expression profiles may be an especially helpful step in studying glioma expression network regarding lncRNAs' crucial regulatory roles
[33]. And furthermore, expression patterns of specific lncRNAs or smaller gene-sets could potentially help us refine glioma subclassification, especially since one of the biggest problems in diagnosis is the morphological similarity of tumours. Indeed, Zhang et al. found novel tumour-related lncRNAs to be associated with malignancy grade and histological differentiation [13], and also our results indicate the existence of measurable differences of individual lncRNA or miRNA expression in glioma, also between histopathological 

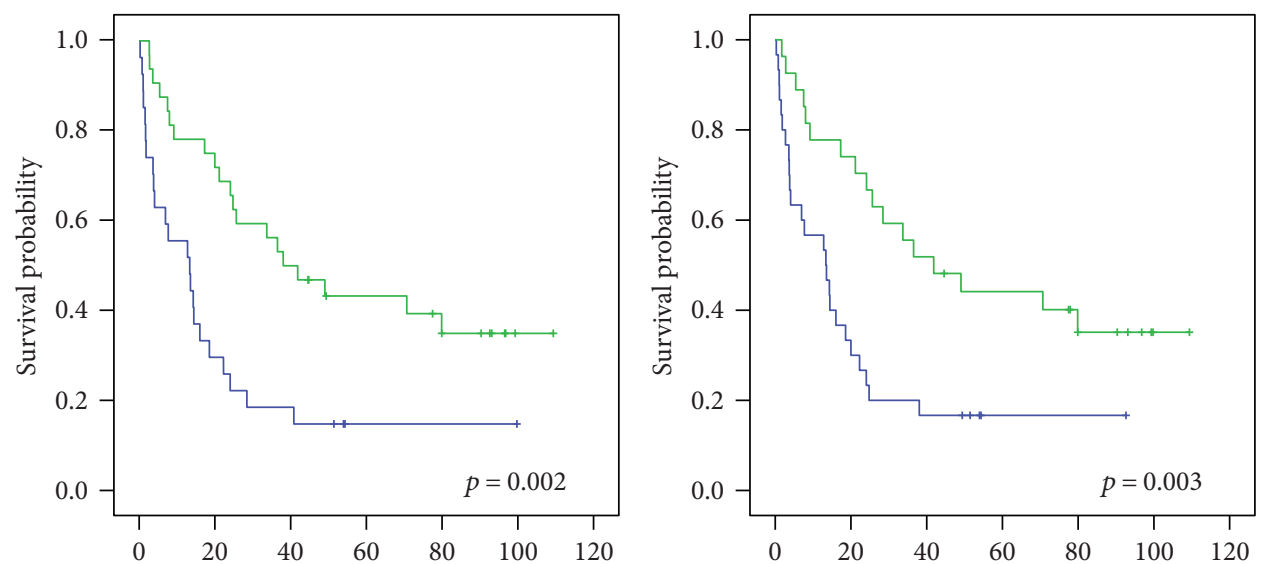

MG3 low/high expression

$\neg$ Low

$\neg$ High

RNCR3 low/high expression

$\neg$ Low

$\neg$ High
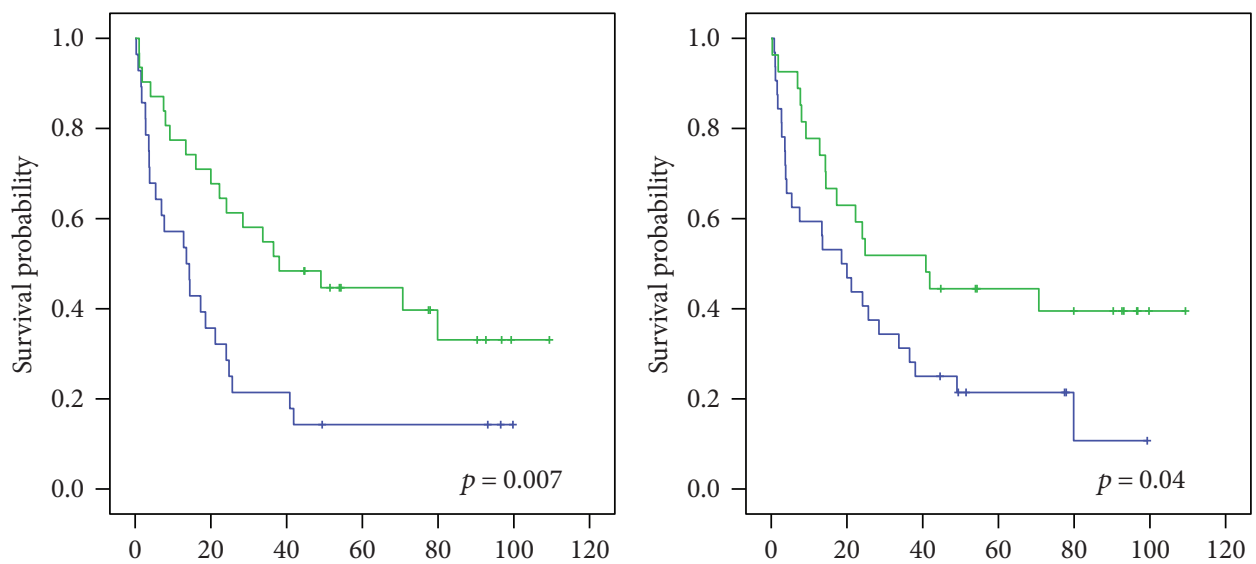

ZFAS1 low/high expression

$\neg$ Low

$\neg$ High

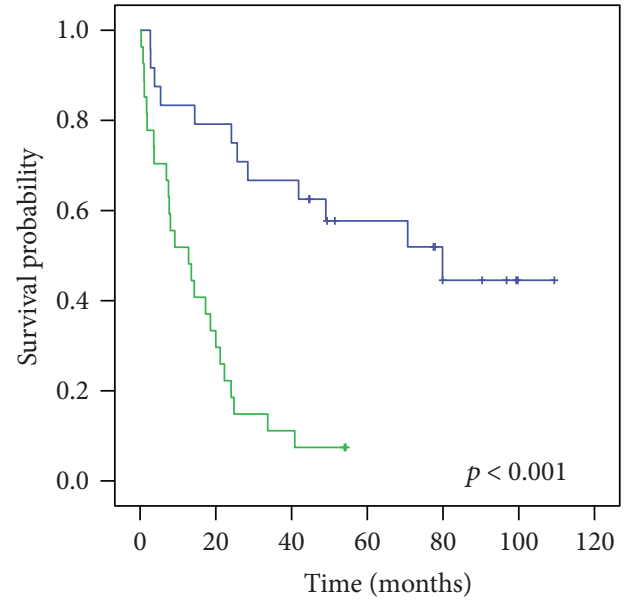

miR-125b low/high expression

$\neg$ Low

$\neg$ High

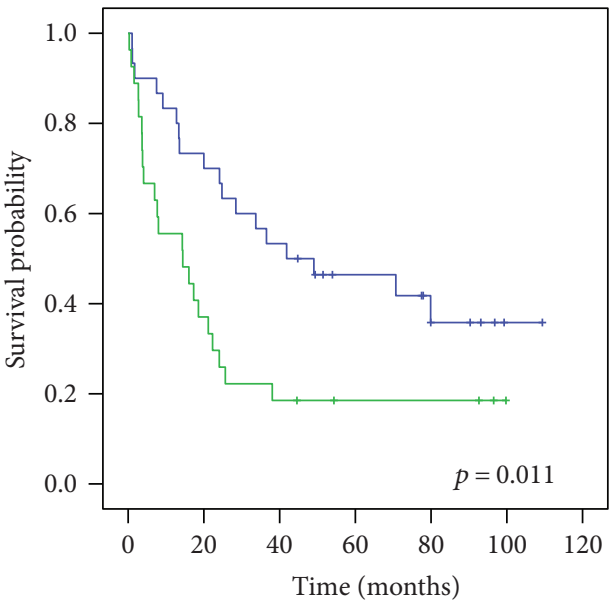

miR-196a low/high expression

miR-770 low/high expression

$\neg$ Low

$\neg$ High

$\neg$ Low

$\neg$ High

FIGURE 8: Expression of lncRNAs and miRNAs shows prognostic significance. Expression of individual sample was classified either low or high regarding the gene's $\Delta \Delta \mathrm{Ct}$ mean expression value. 
subtypes and malignancy grades. Last but not the least, numerous lncRNAs show prognostic value in different human cancer types, including glioma [34-38]. Just recently, but in contrast to our findings, Gao et al. found elevated expression of ZFAS1 in glioma as an independent, unfavourable prognostic factor [39]. Similarly, increased expression of NEAT1 [40], HOTAIR [41], and MALAT1 [23, 38] was also found associated with poor prognosis in glioma. Zhang et al. explored a six-lncRNA signature as a set of prognostic genes in glioma-PART1, MGC21881, MIAT, GAS5, and $P A R 5$ were correlated with prolonged survival and KIAA0495 in association with the poorer [34].

Among seven validated lncRNAs, there is already a considerable research and data for roles of HOTAIR and MEG3 in various cancer types [31, 42-52]. And just recently, Gao et al. reported ZFAS1's oncogenic potential in glioma tumours [39]. IncRNAs can exhibit oncogenic or tumour suppressive functions [11]. Regarding processes of tumour growth, all three were found to be involved in cell proliferation in glioma $[39,53-55]$ and various other cancer types [56-61]. As an oncogenic lncRNA, HOTAIR is involved in epigenetic gene silencing, cell growth, and progression of various cancers [42-44]. A number of studies show that increased expression of HOTAIR enhances cell proliferation and invasion of cancer cells and has been associated with tumour progression and prognosis [45, 55, 56, 59, 62]. Overall high expression of HOTAIR in our glioma samples indicates its association also with glioma development and was already found essential for glioblastoma proliferation $[55,62,63]$. Expression of HOTAIR has been found to differentiate between astrocytoma, oligodendroglioma, and/or oligoastrocytoma, suggesting its use as a potential biomarker in distinguishing morphologically similar cases [41].

On the contrary, MEG3 expression has been strongly decreased in glioma and its expression decreases with malignancy grade, thus acting as a tumour suppressor and contributing to glioma progression [18, 64]. As a putative tumour suppressor, it inhibits cell proliferation/ DNA synthesis by stimulating the expression of tumour suppressor p53 [65] and modulating the binding of p53 on the promoter of its target genes [66]. On the other hand, MEG3 is also able to inhibit cell proliferation and promote cell apoptosis in the p53-independent manner [64]. In line with the latter, its decreased levels are also associated with poorer survival. Overexpression of MEG3 in human glioma cell lines inhibits cell proliferation and promotes cell apoptosis $[18,64]$.

Also found to be involved in p53-dependent cell cycle control is ZFAS1 [54], an antisense lncRNA localized at the $5^{\prime}$ end of the protein-coding gene ZNFX1 [67] that is widely expressed in numerous tissues, including the brain (http:// www.proteinatlas.org). In their research of ZFAS1 in glioma tissues and cell lines, Lv et al. [68] and Gao et al. [39] found its increased expression correlates with tumour stage and poor survival, matching with our results. Significantly increased ZFAS1 expression indicates its oncogenic function [54] -its silencing decreases cell's proliferation through G1 cell cycle arrest [68]. Moreover, by regulating the epithelialmesenchymal transition (EMT) and Notch signalling pathway, it could contribute to glioma progression [39]. In addition to IncRNA's oncogenic or tumour suppressive effect, one can also exhibit both effects [3, 5, 11]. ZFAS1 was found downregulated in breast carcinoma, which suggests ZFAS1 also as a possible tumour suppressor [67].

On the contrary to abovementioned lncRNAs, only little or none is known about the expression and function of lncRNAs EGO-A, RNCR3, JPX, and 7SL in glioma. Abdelmohsen et al. reported $7 S L$ to be highly expressed in various cancer tissues and by repressing $p 53$ mRNA translation consequently promoting cancer cell growth [69]. 7SL is a small RNA component of cytoplasmic SRP (signal recognition particle) complex, a guide that directs nascent secretory proteins towards the endoplasmic reticulum and is necessary for synthesis of normal, active proteins. It is essential for translocation across the membrane of endoplasmic reticulum [70], and its dysregulation may possibly result in impaired protein synthesis machinery. Thus, it is not surprising that $7 S L$ is ubiquitously expressed in numerous tissues, including the brain [71]. Our study showed decreased 7SL levels in astrocytic tumours and increased levels in oligodendroglioma and oligoastrocytoma. These expression differences between oligodendroglioma and astrocytoma may be an additional genetic parameter for distinguishing these subtypes among each other, especially since expression is near normal levels in oligodendroglioma (and oligoastrocytoma).

Expression levels of $E G O-A$ (eosinophil granule ontogeny isoform A) were significantly increased in all glioma subtypes, with the exception of secondary GBMs, which can be probably attributed to the small sample number. It is worth mentioning that secondary GBMs are relatively rare, partly because precursor low-grade or anaplastic astrocytoma develop at younger age than other GBMs, and some patients succumb before the disease progresses, and partly because they are mistakenly classified as primary GBMs [72]. EGO$A$ function is not yet known [73], but its potential role in glioma biogenesis may be implied by the chromosomal location, since its host gene ITPR1 (inositol triphosphate receptor type 1) is encoded in close proximity to EGR-1 (early growth response 1), a transcriptional regulator of genes required for induction of mitosis, cell differentiation, and growth [74]. Expression of EGR-1 gene in glioma cells is induced by overexpression of EGFR and PDGFR genes, thus suggesting $E G R-1$ as a connection of growth factor stimulation with gene expression changes [74]. Association of EGR-1 gene with expression of growth factor might also explain the differences in expression level of EGO-A between the two GBM subtypes (small sample number aside), since EGFR overexpression is typical for primary GBMs, but is rare in secondary GBMs [72]. However, whether the correlation between expression levels of EGR-1 and EGO-A loci exists is yet to be determined. $\mathrm{Xu}$ et al. found expression of $E G O$ transcript to be downregulated in breast cancer and to play an important role in progression of breast cancer and prognosis, thus serving as potential prognostic target [75].

RNCR3 (retinal noncoding RNA 3) is highly expressed in the brain, yet we have not found any reported studies of its expression in glioma, and to date very little is known about its biological function. Especially, it was identified as 
a precursor of the miR-124a, the most abundant miRNA in the vertebrate CNS and necessary for normal brain development [76]. Expression level of $m i R-124 a$ was significantly decreased in anaplastic astrocytoma and GBMs, when compared to normal brain tissue [77], and also in oligodendroglioma [78]. We found substantially decreased expression of $m i R-124 a$ and $R N C R 3$ in primary and secondary GBMs and a positive correlation between expression of these two ncRNAs. Since the RNCR3 is a precursor for $m i R-124 a$, decreased expression of $m i R$ $124 a$ could be a possible consequence of reduced RNCR3 expression. Low expression of RNCR3 also indicates lower survival probability. Regarding the findings, we can infer that RNCR3 and its encoded $m i R-124 a$ may be implicated in the development and progression of glioma. $m i R-124 a$ is the most extensively investigated miRNAs in glioma cell proliferation. In the earliest study, it has been shown that upregulation of miR-124a induces glioblastoma cell cycle arrest. These results suggested that targeted delivery of miR-124 to glioblastoma tumour cells may be therapeutically efficacious for the treatment of this disease [77]. Fowler et al. [79] found an ectopic expression of $m i R-$ $124 a$ significantly inhibiting GBM migration and invasion, which once more supports its role in glioma progression. Moreover, restoration of $m i R-124 a$ could inhibit glioma cell proliferation and invasion through $m i R-124 a$ blocking the expression of the IQGAP1 gene and downstream $\beta$ catenin and cyclin D1 [80] and PIM1 in astrocytoma cancer cells [81]. Additionally, transfection with $m i R-124$ inhibitor rescued the proliferative ability of human glioma cells. Results demonstrated that miR-124 is an important downstream target gene of Hedgehog signalling and that glioma-associated oncogene-miR-124-AURKA axis is essential for the proliferation and growth of human glioma cells [82].

Expression of miR-770 showed decreased levels in lower malignancy grades and a better survival probability. The expression was in an inverse correlation to its host gene, that is, MEG3, and regarding the findings of miR-770 being a downstream transcriptional target of $\mathrm{Wnt} / \beta$-signalling pathway [83], it is reasonable to propose for MEG3 and miR-770 possibly having different mechanisms regulating their expression. There are only few investigations performed about the role of $m i R-770$ in carcinogenesis; moreover, only one study is investigating its role in proliferation. By acting as a sponge for $m i R-770$, leading to its downregulation, lncRNA PCGEM1 stimulates proliferation of osteoarthritic synoviocytes [84]. Whether or not this is also the case in glioma cells is yet to be investigated. Analyses of miRNAlncRNA-mRNA interactions using their expression profiles alone are not enough to further understand the potential relationships between different RNA molecules [85]. Differences in IncRNA and its associated miRNAs' expression can also be the consequence of different stability of the transcript, splicing, promoter methylation, or miRNA's maturation [26].

miR-196a is concurrently upregulated with HOTAIR in gastrointestinal stromal tumours (GISTs) [31], but was downregulated in our glioma subtypes, with no association to HOTAIR. It has been shown that $m i R-196 a$ is expressed in lower levels in oligodendrogliomas, astrocytomas, and oligoastrocytomas compared to glioblastoma, similar to our results [86]. $m i R-196 a$ expression seems to be highly associated with patient's survival as its higher expression indicates poorer prognosis and shorter survival time, coinciding with HOTAIR overexpression survival prediction and their suggested coregulated expression [41]. Investigating the oncogenic effects of miR-196a in vivo and in vitro revealed that it induces and promotes proliferation and suppresses apoptosis through inhibition of the $\mathrm{I} \kappa \mathrm{B} \alpha$ [87].

We were not able to detect any significant correlation between expression patterns of $m i R-125 b$ and $7 S L$, although there is an inverse overall expression in oligoastrocytoma, oligodendroglioma, and secondary GBMs. $m i R-125 b$ has already been shown to be upregulated in primary GBMs (but downregulated in GBM cell lines) [88] and in oligodendroglioma [78]. Its overexpression promotes glioma cell proliferation and inhibits cell apoptosis, thus supporting the suggested oncogenic role $[89,90]$.

\section{Conclusions}

Studies of lncRNAs and miRNAs over the past decade increasingly reveal the importance of ncRNAs in cancer development and progression. All in all, our results corroborate the involvement of noncoding RNAs in the complex nature of primary brain tumours. We show that several lncRNAs, some already related to glioma and some investigated for the first time, could importantly contribute to glioma development. Expression level of several lncRNAs and certain lncRNA-associated miRNAs significantly changes between the analysed glioma subtypes and malignancy grades. Moreover, their possible correlation with miRNAs suggests a complex interplay of lncRNA-miRNA in regulating gene expression in glioma. Observing high expressional variability of the results opens numerous further questions and presents the foundation for further research of noncoding RNA implication in glioma formation and development, which will conceivably provide new glioma-specific biomarkers and targets for treatment of the disease. Although our research does not show/determine the exact mechanism of ncRNAs in tumour development, it does show that the analyses of expressional screening represent guidelines for further research and are efficient in the search of new cancer-related genes. It has been already accepted that lncRNAs have an immense role in glioma pathophysiology, but the question is which lncRNAs are implicated in malignant transformation and how they contribute to each level of tumorigenesis.

\section{Ethical Approval}

The study was approved by the Slovene National Ethics Committee and was therefore performed in accordance with the ethical standards, laid down in the 1964 Declaration of Helsinki and its later amendments. Informed consent of the patient for conducting the research was not necessary. 


\section{Disclosure}

The funding organization had no role in the study design, data collection and analysis, decision to publish, or preparation of the manuscript.

\section{Conflicts of Interest}

All authors certify that they have no affiliations with or involvement in any organization or entity with any financial interest (such as honoraria; educational grants; participation in speakers and bureaus; membership, employment, consultancies, stock ownership, or other equity interest; and expert testimony or patent-licensing arrangements) or nonfinancial interest (such as personal or professional relationships, affiliations, knowledge, or beliefs) in the subject matter or materials discussed in this manuscript.

\section{Authors' Contributions}

Alenka Matjašič, Mojca Tajnik, Emanuela Boštjančič, and Damjan Glavač contributed in the conception and design of the study. Boštjan Matos and Mara Popović performed the acquisition and diagnosis of glioma specimens. Alenka Matjašič, Emanuela Boštjančič, and Mojca Tajnik contributed in the development of the methodology and performed the experiment. Alenka Matjašič and Emanuela Boštjančič performed the analysis and interpretation of data. Alenka Matjašič, Mojca Tajnik, Emanuela Boštjančič, Mara Popović, Boštjan Matos, and Damjan Glavač were responsible for the writing, review, and/or revision of the manuscript. All authors have read and approved the manuscript. Alenka Matjašič, Mojca Tajnik, and Emanuela Boštjančič contributed equally to this work.

\section{Acknowledgments}

This research was supported by the Slovenian Research Agency (grants to Alenka Matjašič and Mojca Tajnik).

\section{References}

[1] M. Esteller, "Non-coding RNAs in human disease," Nature Reviews Genetics, vol. 12, pp. 861-874, 2011.

[2] J. L. Rinn and H. Y. Chang, "Genome regulation by long noncoding RNAs," Annual Review of Biochemistry, vol. 81, pp. 145-166, 2012.

[3] X. Li, Z. Wu, X. Fu, and W. Han, "Long noncoding RNAs: insights from biological features and functions to diseases," Medicinal Research Reviews, vol. 33, pp. 517-553, 2013.

[4] I. A. Qureshi and M. F. Mehler, "Emerging roles of non-coding RNAs in brain evolution, development, plasticity and disease," Nature Reviews Neuroscience, vol. 13, pp. 528-541, 2012.

[5] M. Huarte and J. L. Rinn, "Large non-coding RNAs: missing links in cancer?," Human Molecular Genetics, vol. 19, pp. R152-R161, 2010.

[6] J. S. Mattick, "The genetic signatures of noncoding RNAs," PLoS Genetics, vol. 5, article e1000459, 2009.
[7] I. A. Qureshi, J. S. Mattick, and M. F. Mehler, "Long noncoding RNAs in nervous system function and disease," Brain Research, vol. 1338, pp. 20-35, 2010.

[8] K. M. Kiang, X. Q. Zhang, and G. K. Leung, "Long non-coding RNAs: the key players in glioma pathogenesis," Cancer, vol. 7, pp. 1406-1424, 2015.

[9] R. Alelu-Paz, N. Ashour, A. Gonzalez-Corpas, and S. Ropero, "DNA methylation, histone modifications, and signal transduction pathways: a close relationship in malignant gliomas pathophysiology," Journal of Signal Transduction, vol. 2012, Article ID 956958, 8 pages, 2012.

[10] G. I. Evan and K. H. Vousden, "Proliferation, cell cycle and apoptosis in cancer," Nature, vol. 411, pp. 342-348, 2001.

[11] E. M. Reis and S. Verjovski-Almeida, "Perspectives of long non-coding RNAs in cancer diagnostics," Frontiers in Genetics, vol. 3, p. 32, 2012.

[12] E. A. Gibb, C. J. Brown, and W. L. Lam, "The functional role of long non-coding RNA in human carcinomas," Molecular Cancer, vol. 10, p. 38, 2011.

[13] X. Zhang, S. Sun, J. K. Pu et al., "Long non-coding RNA expression profiles predict clinical phenotypes in glioma," Neurobiology of Disease, vol. 48, pp. 1-8, 2012.

[14] S. D. Fouse and J. F. Costello, "Epigenetics of neurological cancers," Future Oncology (London, England), vol. 5, pp. 1615-1629, 2009.

[15] D. E. Handy, R. Castro, and J. Loscalzo, "Epigenetic modifications: basic mechanisms and role in cardiovascular disease," Circulation, vol. 123, pp. 2145-2156, 2011.

[16] Y. K. Kim and V. N. Kim, "Processing of intronic micro RNAs,” The EMBO Journal, vol. 26, pp. 775-783, 2007.

[17] E. A. Gibb, E. A. Vucic, K. S. Enfield et al., "Human cancer long non-coding RNA transcriptomes," PLoS One, vol. 6, article e25915, 2011.

[18] P. Wang, Z. Ren, and P. Sun, "Overexpression of the long noncoding RNA MEG3 impairs in vitro glioma cell proliferation," Journal of Cellular Biochemistry, vol. 113, pp. 1868-1874, 2012.

[19] E. Hulleman and K. Helin, "Molecular mechanisms in gliomagenesis," Advances in Cancer Research, vol. 94, pp. 1-27, 2005.

[20] J. Y. Tang, J. C. Lee, Y. T. Chang et al., "Long noncoding RNAs-related diseases, cancers, and drugs," The Scientific World Journal, vol. 2013, Article ID 943539, 7 pages, 2013.

[21] M. Pojo and C. B. Marques, "Molecular hallmarks of gliomas," in Molecular Targets of CNS Tumors, dM Garami, Ed., pp. 177-200, InTech, Croatia, 2011.

[22] X. Shi, M. Sun, H. Liu, Y. Yao, and Y. Song, "Long non-coding RNAs: a new frontier in the study of human diseases," Cancer Letters, vol. 339, pp. 159-166, 2013.

[23] K. X. Ma, H. J. Wang, X. R. Li et al., "Long noncoding RNA MALAT1 associates with the malignant status and poor prognosis in glioma," Tumour Biology: the Journal of the International Society for Oncodevelopmental Biology and Medicine, vol. 36, pp. 3355-3359, 2015.

[24] H. Guo, L. Wu, Q. Yang, M. Ye, and X. Zhu, "Functional linc-POU3F3 is overexpressed and contributes to tumorigenesis in glioma," Gene, vol. 554, pp. 114-119, 2015.

[25] H. Rothenmund, H. Singh, B. Candas et al., "Hereditary colorectal cancer registries in Canada: report from the Colorectal Cancer Association of Canada consensus meeting; Montreal, Quebec; October 28, 2011," Current Oncology (Toronto, Ont), vol. 20, pp. 273-278, 2013. 
[26] L. F. Gulyaeva and N. E. Kushlinskiy, "Regulatory mechanisms of microRNA expression," Journal of Translational Medicine, vol. 14, p. 143, 2016.

[27] C. L. Andersen, J. L. Jensen, and T. F. Orntoft, "Normalization of real-time quantitative reverse transcription-PCR data: a model-based variance estimation approach to identify genes suited for normalization, applied to bladder and colon cancer data sets," Cancer Research, vol. 64, pp. 5245-5250, 2004.

[28] K. J. Livak and T. D. Schmittgen, "Analysis of relative gene expression data using real-time quantitative PCR and the 2(-Delta Delta C(T)) method," Methods (San Diego, Calif), vol. 25, pp. 402-408, 2001.

[29] S. Jalali, D. Bhartiya, M. K. Lalwani, S. Sivasubbu, and V. Scaria, "Systematic transcriptome wide analysis of lncRNAmiRNA interactions," PLoS One, vol. 8, article e53823, 2013.

[30] X. Wei, D. Chen, T. Lv, G. Li, and S. Qu, "Serum microRNA$125 \mathrm{~b}$ as a potential biomarker for glioma diagnosis," Molecular Neurobiology, vol. 53, pp. 163-170, 2014.

[31] T. Niinuma, H. Suzuki, M. Nojima et al., "Upregulation of miR-196a and HOTAIR drive malignant character in gastrointestinal stromal tumors," Cancer Research, vol. 72, pp. 11261136, 2012.

[32] P. P. Amaral, M. B. Clark, D. K. Gascoigne, M. E. Dinger, and J. S. Mattick, "lncRNAdb: a reference database for long noncoding RNAs," Nucleic Acids Research, vol. 39, pp. D146-D151, 2011.

[33] Q. Liao, C. Liu, X. Yuan et al., "Large-scale prediction of long non-coding RNA functions in a coding-non-coding gene co-expression network," Nucleic Acids Research, vol. 39, pp. 3864-3878, 2011.

[34] X. Q. Zhang, S. Sun, K. F. Lam et al., "A long noncoding RNA signature in glioblastoma multiforme predicts survival," Neurobiology of Disease, vol. 58, pp. 123-131, 2013.

[35] Z. Du, T. Fei, R. G. Verhaak et al., "Integrative genomic analyses reveal clinically relevant long noncoding RNAs in human cancer," Nature Structural \& Molecular Biology, vol. 20, pp. 908-913, 2013.

[36] Y. Sun, Z. Wang, and D. Zhou, "Long non-coding RNAs as potential biomarkers and therapeutic targets for gliomas," Medical Hypotheses, vol. 81, pp. 319-321, 2013.

[37] M. Sun, F. Y. Jin, R. Xia et al., "Decreased expression of long noncoding RNA GAS5 indicates a poor prognosis and promotes cell proliferation in gastric cancer," BMC Cancer, vol. 14, p. 319, 2014.

[38] M. Sun, R. Xia, F. Jin et al., "Downregulated long noncoding RNA MEG3 is associated with poor prognosis and promotes cell proliferation in gastric cancer," Tumour Biology: the Journal of the International Society for Oncodevelopmental Biology and Medicine, vol. 35, pp. 1065-1073, 2014.

[39] K. Gao, Z. Ji, K. She, Q. Yang, and L. Shao, "Long non-coding RNA ZFAS1 is an unfavourable prognostic factor and promotes glioma cell progression by activation of the Notch signaling pathway," Biomedicine \& Pharmacotherapy = Biomedecine \& Pharmacotherapie, vol. 87, pp. 555-560, 2017.

[40] C. He, B. Jiang, J. Ma, and Q. Li, “Aberrant NEAT1 expression is associated with clinical outcome in high grade glioma patients," APMIS: Acta Pathologica, Microbiologica, et Immunologica Scandinavica, vol. 124, pp. 169-174, 2016.

[41] J. X. Zhang, L. Han, Z. S. Bao et al., "HOTAIR, a cell cycleassociated long noncoding RNA and a strong predictor of survival, is preferentially expressed in classical and mesenchymal glioma," Neuro-Oncology England, vol. 15, pp. 1595-1603, 2013.

[42] R. A. Gupta, N. Shah, K. C. Wang et al., "Long non-coding RNA HOTAIR reprograms chromatin state to promote cancer metastasis," Nature, vol. 464, pp. 1071-1076, 2010.

[43] R. Kogo, T. Shimamura, K. Mimori et al., "Long noncoding RNA HOTAIR regulates polycomb-dependent chromatin modification and is associated with poor prognosis in colorectal cancers," Cancer Research, vol. 71, pp. 6320-6326, 2011.

[44] X. B. Lv, G. Y. Lian, H. R. Wang, E. Song, H. Yao, and M. H. Wang, "Long noncoding RNA HOTAIR is a prognostic marker for esophageal squamous cell carcinoma progression and survival," PLoS One, vol. 8, article e63516, 2013.

[45] B. Cai, X. Q. Song, J. P. Cai, and S. Zhang, "HOTAIR: a cancerrelated long non-coding RNA,” Neoplasma, vol. 61, pp. 379391, 2014.

[46] X. Zhang, Y. Zhou, K. R. Mehta et al., "A pituitary-derived MEG3 isoform functions as a growth suppressor in tumor cells," The Journal of Clinical Endocrinology and Metabolism, vol. 88, pp. 5119-5126, 2003.

[47] C. Braconi, T. Kogure, N. Valeri et al., "microRNA-29 can regulate expression of the long non-coding RNA gene MEG3 in hepatocellular cancer," Oncogene, vol. 30, pp. 4750-4756, 2011.

[48] K. H. Lu, W. Li, X. H. Liu et al., "Long non-coding RNA MEG3 inhibits NSCLC cells proliferation and induces apoptosis by affecting p53 expression,” BMC Cancer, vol. 13, p. 461, 2013.

[49] R. Qin, Z. Chen, Y. Ding, J. Hao, J. Hu, and F. Guo, "Long noncoding RNA MEG3 inhibits the proliferation of cervical carcinoma cells through the induction of cell cycle arrest and apoptosis," Neoplasma, vol. 60, pp. 486-492, 2013.

[50] L. Ying, Y. Huang, H. Chen et al., "Downregulated MEG3 activates autophagy and increases cell proliferation in bladder cancer," Molecular BioSystems, vol. 9, pp. 407-411, 2013.

[51] G. Luo, M. Wang, X. Wu et al., "Long non-coding RNA MEG3 inhibits cell proliferation and induces apoptosis in prostate cancer," Cellular Physiology and Biochemistry: International Journal of Experimental Cellular Physiology, Biochemistry, and Pharmacology, vol. 37, pp. 2209-2220, 2015.

[52] J. Li, E. B. Bian, X. J. He et al., "Epigenetic repression of long non-coding RNA MEG3 mediated by DNMT1 represses the p53 pathway in gliomas," International Journal of Oncology, vol. 48, pp. 723-733, 2016.

[53] Y. Chen, Y. Bian, S. Zhao, F. Kong, and X. Li, "Suppression of PDCD4 mediated by the long non-coding RNA HOTAIR inhibits the proliferation and invasion of glioma cells," Oncology Letters, vol. 12, pp. 5170-5176, 2016.

[54] N. Thorenoor, P. Faltejskova-Vychytilova, S. Hombach et al., "Long non-coding RNA ZFAS1 interacts with CDK1 and is involved in p53-dependent cell cycle control and apoptosis in colorectal cancer," Oncotarget, vol. 7, no. 1, pp. 622-637, 2016.

[55] K. Zhang, X. Sun, X. Zhou et al., "Long non-coding RNA HOTAIR promotes glioblastoma cell cycle progression in an EZH2 dependent manner," Oncotarget United States, vol. 6, pp. 537-546, 2015.

[56] S. N. Min, T. Wei, X. T. Wang, L. L. Wu, and G. Y. Yu, "Clinicopathological and prognostic significance of homeobox transcript antisense RNA expression in various cancers: a meta-analysis," Medicine, vol. 96, article e7084, 2017. 
[57] W. Guo, Z. Dong, S. Liu et al., "Promoter hypermethylationmediated downregulation of miR-770 and its host gene MEG3, a long non-coding RNA, in the development of gastric cardia adenocarcinoma," Molecular Carcinogenesis, vol. 56, 2017.

[58] W. P. Chak, R. W. Lung, J. H. Tong et al., "Downregulation of long non-coding RNA MEG3 in nasopharyngeal carcinoma," Molecular Carcinogenesis, vol. 56, pp. 1041-1054, 2017.

[59] Y. Yu, F. Lv, D. Liang et al., "HOTAIR may regulate proliferation, apoptosis, migration and invasion of MCF-7 cells through regulating the P53/Akt/JNK signaling pathway," Biomedicine \& Pharmacotherapy = Biomedecine \& Pharmacotherapie, vol. 90, pp. 555-561, 2017.

[60] L. Pan, W. Liang, M. Fu et al., "Exosomes-mediated transfer of long noncoding RNA ZFAS1 promotes gastric cancer progression," Journal of Cancer Research and Clinical Oncology, vol. 143, pp. 991-1004, 2017.

[61] F. Nie, X. Yu, M. Huang et al., "Long noncoding RNA ZFAS1 promotes gastric cancer cells proliferation by epigenetically repressing KLF2 and NKD2 expression," Oncotarget, vol. 8, pp. 38227-38238, 2017.

[62] X. Zhou, Y. Ren, J. Zhang et al., "HOTAIR is a therapeutic target in glioblastoma," Oncotarget, vol. 6, pp. 8353-8365, 2015.

[63] C. Pastori, P. Kapranov, C. Penas et al., "The bromodomain protein BRD4 controls HOTAIR, a long noncoding RNA essential for glioblastoma proliferation," Proceedings of the National Academy of Sciences of the United States of America, vol. 112, pp. 8326-8331, 2015.

[64] Y. Zhou, X. Zhang, and A. Klibanski, "MEG3 noncoding RNA: a tumor suppressor," Journal of Molecular Endocrinology, vol. 48, pp. R45-R53, 2012.

[65] X. Zhang, K. Rice, Y. Wang et al., "Maternally expressed gene 3 (MEG3) noncoding ribonucleic acid: isoform structure, expression, and functions," Endocrinology, vol. 151, pp. 939947, 2010.

[66] M. U. Kaikkonen, M. T. Lam, and C. K. Glass, "Non-coding RNAs as regulators of gene expression and epigenetics," Cardiovascular Research, vol. 90, pp. 430-440, 2011.

[67] M. E. Askarian-Amiri, J. Crawford, J. D. French et al., "SNORD-host RNA Zfas1 is a regulator of mammary development and a potential marker for breast cancer," RNA (New York, NY), vol. 17, pp. 878-891, 2011.

[68] Q. L. Lv, S. H. Chen, X. Zhang et al., "Upregulation of long noncoding RNA zinc finger antisense 1 enhances epithelialmesenchymal transition in vitro and predicts poor prognosis in glioma," Tumour Biology: the Journal of the International Society for Oncodevelopmental Biology and Medicine, vol. 39, article 1010428317695022, 2017.

[69] K. Abdelmohsen, A. C. Panda, M. J. Kang et al., "7SL RNA represses p53 translation by competing with HuR," Nucleic Acids Research, vol. 42, pp. 10099-10111, 2014.

[70] C. Zwieb, R. W. van Nues, M. A. Rosenblad, J. D. Brown, and T. Samuelsson, "A nomenclature for all signal recognition particle RNAs,” RNA (New York, NY), vol. 11, pp. 7-13, 2005.

[71] J. C. Castle, C. D. Armour, M. Löwer et al., "Digital genomewide ncRNA expression, including SnoRNAs, across 11 human tissues using polyA-neutral amplification," PLoS One, vol. 5, article e11779, 2010.

[72] H. Ohgaki and P. Kleihues, "The definition of primary and secondary glioblastoma," Clinical Cancer Research: an Official
Journal of the American Association for Cancer Research, vol. 19, pp. 764-772, 2013.

[73] D. Rose and P. F. Stadler, "Molecular evolution of the noncoding eosinophil granule ontogeny transcript," Frontiers in Genetics, vol. 2, p. 69, 2011.

[74] K. Kaufmann and G. Thiel, "Epidermal growth factor and platelet-derived growth factor induce expression of Egr-1, a zinc finger transcription factor, in human malignant glioma cells," Journal of the Neurological Sciences, vol. 189, pp. 83-91, 2001.

[75] S. P. Xu, J. F. Zhang, S. Y. Sui et al., "Downregulation of the long noncoding RNA EGOT correlates with malignant status and poor prognosis in breast cancer," Tumour Biology: the Journal of the International Society for Oncodevelopmental Biology and Medicine, vol. 36, pp. 9807-9812, 2015.

[76] R. Sanuki, A. Onishi, C. Koike et al., "miR-124a is required for hippocampal axogenesis and retinal cone survival through Lhx2 suppression," Nature Neuroscience, vol. 14, pp. 1125$1134,2011$.

[77] J. Silber, D. A. Lim, C. Petritsch et al., "miR-124 and miR-137 inhibit proliferation of glioblastoma multiforme cells and induce differentiation of brain tumor stem cells," BMC Medicine, vol. 6, p. 14, 2008.

[78] P. T. Nelson, D. A. Baldwin, W. P. Kloosterman, S. Kauppinen, R. H. Plasterk, and Z. Mourelatos, "RAKE and LNA-ISH reveal microRNA expression and localization in archival human brain," RNA (New York, NY), vol. 12, pp. 187-191, 2006.

[79] A. Fowler, D. Thomson, K. Giles et al., "miR-124a is frequently down-regulated in glioblastoma and is involved in migration and invasion," European Journal of Cancer, vol. 47, pp. 953963, 2011.

[80] S. H. Lu, X. J. Jiang, G. L. Xiao, D. Y. Liu, and X. R. Yuan, "miR-124a restoration inhibits glioma cell proliferation and invasion by suppressing IQGAP1 and $\beta$-catenin," Oncology Reports, vol. 32, pp. 2104-2110, 2014.

[81] D. Deng, L. Wang, Y. Chen et al., "MicroRNA-124-3p regulates cell proliferation, invasion, apoptosis, and bioenergetics by targeting PIM1 in astrocytoma," Cancer Science, vol. 107, pp. 899-907, 2016.

[82] L. Xu, H. Liu, Z. Yan, Z. Sun, S. Luo, and Q. Lu, "Inhibition of the Hedgehog signaling pathway suppresses cell proliferation by regulating the Gli2/miR-124/AURKA axis in human glioma cells," International Journal of Oncology, vol. 50, pp. 18681878, 2017.

[83] W. J. Wu, J. Shi, G. Hu et al., "Wnt/ $\beta$-catenin signaling inhibits FBXW7 expression by upregulation of microRNA-770 in hepatocellular carcinoma," Tumour Biology: the Journal of the International Society for Oncodevelopmental Biology and Medicine, vol. 37, pp. 6045-6051, 2016.

[84] Y. Kang, J. Song, D. Kim et al., "PCGEM1 stimulates proliferation of osteoarthritic synoviocytes by acting as a sponge for miR-770," Journal of Orthopaedic Research: Official Publication of the Orthopaedic Research Society, vol. 34, pp. 412$418,2016$.

[85] L. Guo, Y. Zhao, S. Yang, H. Zhang, and F. Chen, "An integrated analysis of miRNA, lncRNA, and mRNA expression profiles," BioMed Research International, vol. 2014, Article ID 345605, 12 pages, 2014.

[86] Y. Guan, M. Mizoguchi, K. Yoshimoto et al., "MiRNA-196 is upregulated in glioblastoma but not in anaplastic astrocytoma and has prognostic significance," Clinical Cancer Research: an 
Official Journal of the American Association for Cancer Research, vol. 16, pp. 4289-4297, 2010.

[87] G. Yang, D. Han, X. Chen et al., "MiR-196a exerts its oncogenic effect in glioblastoma multiforme by inhibition of IkappaBalpha both in vitro and in vivo," Neuro-Oncology, vol. 16, pp. 652-661, 2014.

[88] S. A. Ciafrè, S. Galardi, A. Mangiola et al., "Extensive modulation of a set of microRNAs in primary glioblastoma," Biochemical and Biophysical Research Communications, vol. 334, pp. 1351-1358, 2005.

[89] X. Li, J. Zheng, L. Chen, H. Diao, and Y. Liu, "Predictive and prognostic roles of abnormal expression of tissue miR-125b, miR-221, and miR-222 in glioma," Molecular Neurobiology, vol. 53, no. 1, pp. 577-583, 2014.

[90] H. F. Xia, T. Z. He, C. M. Liu et al., "MiR-125b expression affects the proliferation and apoptosis of human glioma cells by targeting Bmf," Cellular Physiology and Biochemistry: International Journal of Experimental Cellular Physiology, Biochemistry, and Pharmacology, vol. 23, pp. 347-358, 2009. 

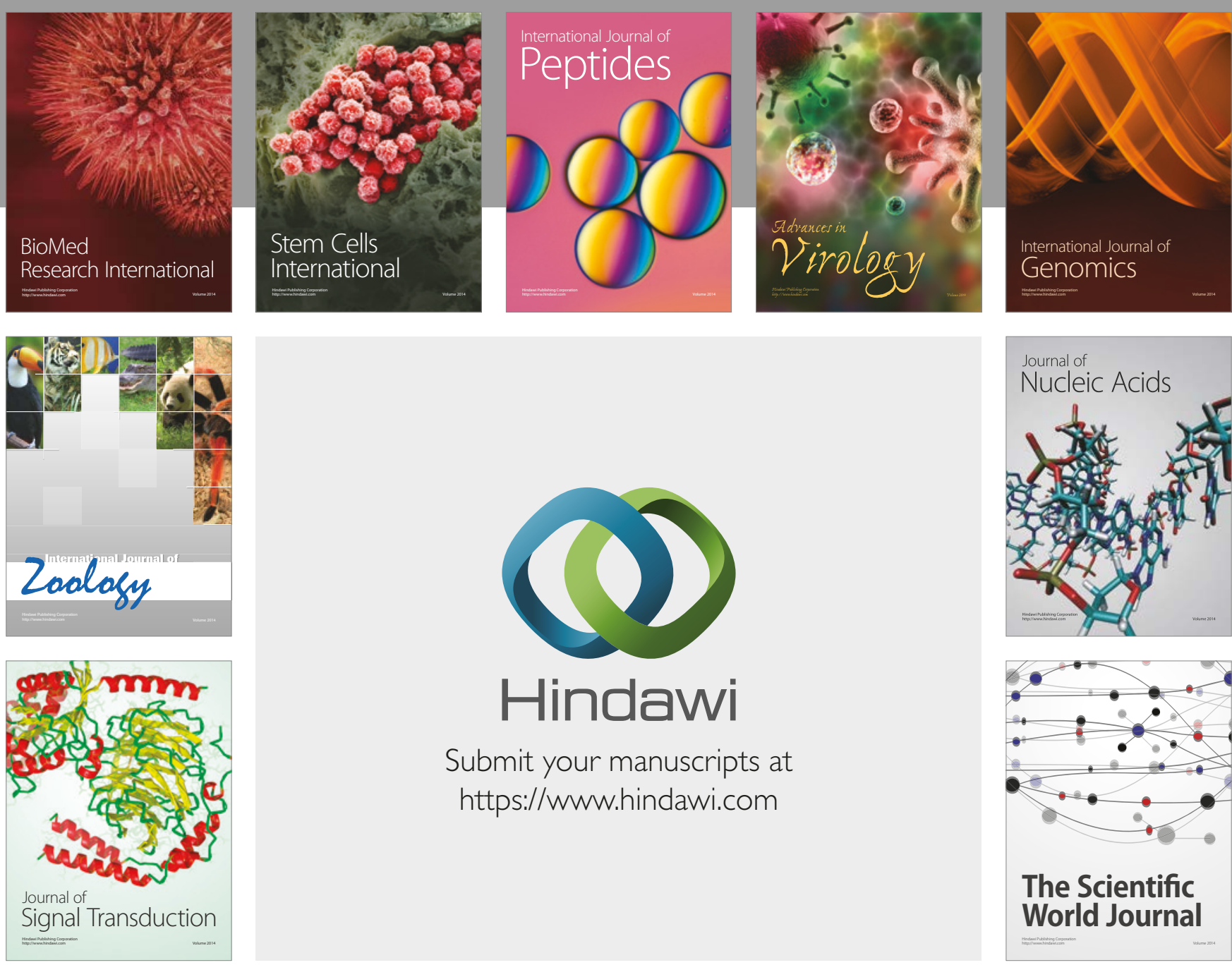

Submit your manuscripts at

https://www.hindawi.com
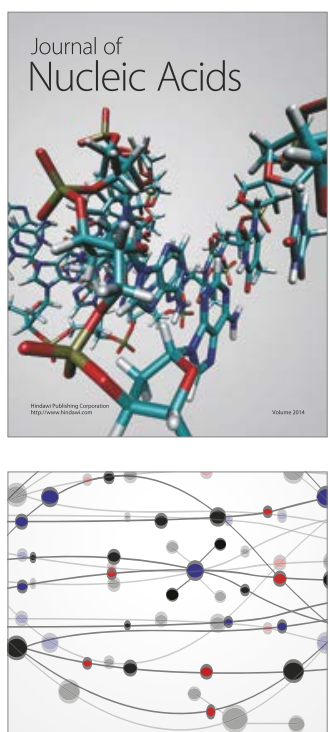

The Scientific World Journal

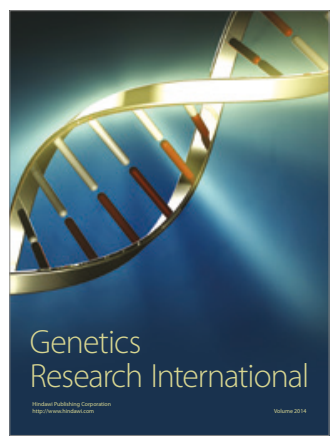

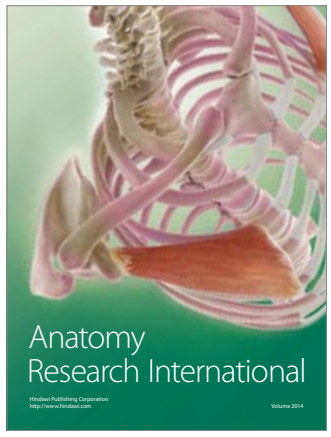

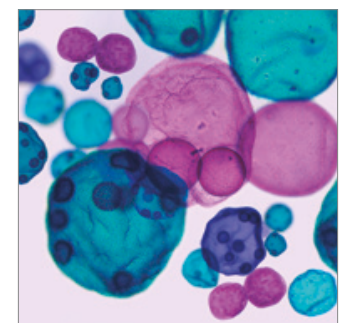

International Journal of Microbiology
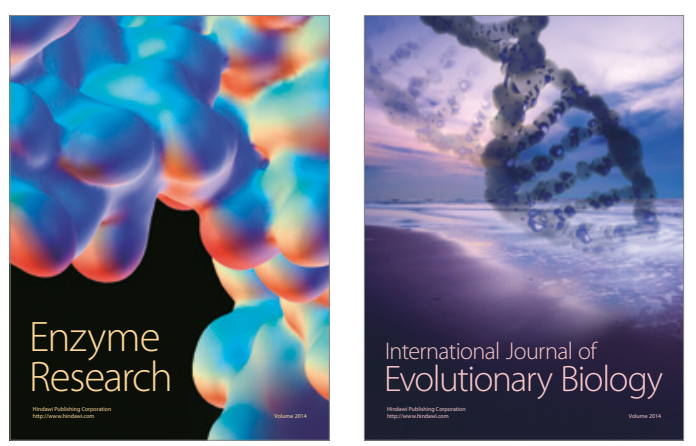
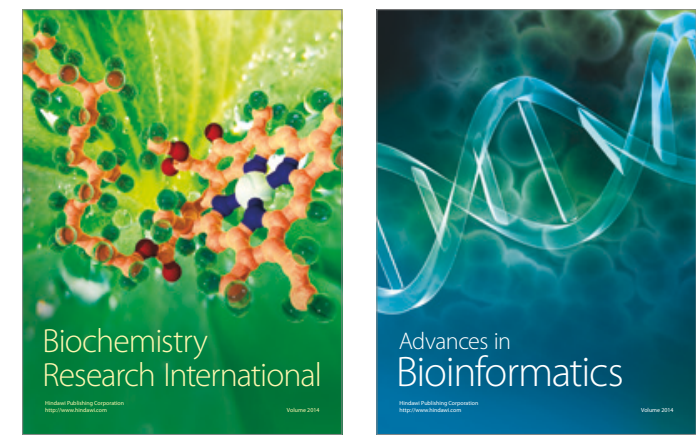

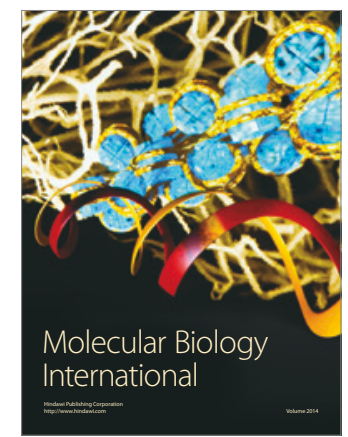

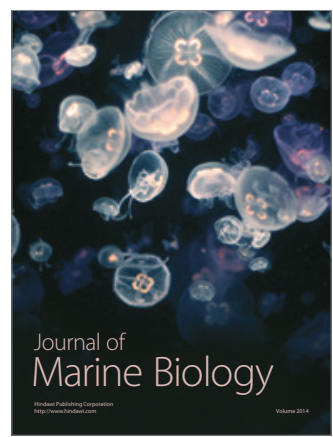

